\title{
2HDM portal for singlet-doublet dark matter
}

\author{
Giorgio Arcadi ${ }^{1, \mathrm{a}}$ \\ ${ }^{1}$ Max-Planck-Institut für Kernphysik (MPIK), Saupfercheckweg 1, 69117 Heidelberg, Germany
}

Received: 27 June 2018 / Accepted: 10 October 2018 / Published online: 26 October 2018

(C) The Author(s) 2018

\begin{abstract}
We present an extensive analysis of a model in which the (Majorana) Dark Matter candidate is a mixture between a $\mathrm{SU}(2)$ singlet and two $\mathrm{SU}(2)$ doublets. This kind of setup takes the name of singlet-doublet model. We will investigate in detail an extension of this model in which the Dark Matter sector interactions with a 2-doublet Higgs sector enforcing the complementarity between Dark Matter phenomenology and searches of extra Higgs bosons.
\end{abstract}

\section{Contents}

1 Introduction . . . . . . . . . . . . . 1

2 The model . . . . . . . . . . . . . . . 2

$2.12 \mathrm{HDM}$ and coupling to the SM . . . . . . . 2

2.2 Coupling to the DM . . . . . . . . . . 3

3 Constraints on the Higgs sector . . . . . . . . . 4

3.1 Bounds on the potential . . . . . . . . . . 4

3.2 EWPT . . . . . . . . . . . . 5 5

3.3 Collider searches of the new Higgs bosons . . . 5

3.4 Limits from flavour . . . . . . . . . . . . 6

3.5 Scanning the parameter space . . . . . . . 6

4 DM constraints . . . . . . . . . . . . . . . . 7

4.1 Relic density . . . . . . . . . . . . . . 7

4.2 Direct detection . . . . . . . . . . . . . . 9

4.3 Indirect detection . . . . . . . . . . . . 10

4.4 Invisible decays of the Higgs and of the Z . . 10

4.5 LHC searches of the new fermions . . . . . 10

4.6 Sommerfeld enhancement for relic density and Indirect Detection . . . . . . . . . . . . . . 11

5 Results and discussion . . . . . . . . . . . . . 12

6 Conclusions ................ . . 13

A Approximate analytical expressions for the DM annihilation cross-section . . . . . . . . . . . . 14

B Couplings of DM with the Higgs bosons . . . . . 15

References . . . . . . . . . . . . . . 16

a e-mail: giorgio.arcadi@mpi-hd.mpg.de

\section{Introduction}

The WIMP paradigm is a compelling solution of the Dark Matter (DM) problem. It relates the achievement of the DM relic density, as measured with unprecedented precision by the PLANCK collaboration [1], to a specific range of values of the thermally averaged pair annihilation cross-section of the DM. An implication of this setup is that the DM should possess sizeable interactions with the Standard Model (SM) particles, making possible a detection at present experimental facilities.

From the model building perspective, a rather simple realization of the interactions needed by the WIMP paradigm consists into the existence of an electrically neutral mediator, coupled with pairs of DM states as well as pairs of SM particles. The Higgs boson is a privileged candidate for this role [2-15].

A fermionic DM candidate, if it is a SM singlet, can couple, in pairs, with the Higgs boson only through $D>4$ operators. ${ }^{1}$ The so called singlet-doublet models $[17-21]^{2}$ overcome this problem by enlarging the spectrum of BSM states by two $S U(2)$ doublets, so that the DM is a mixture of their neutral components as well as of the singlet originally introduced. This has, however, the consequence that the $\mathrm{DM}$ can interact, in pairs, also with the $Z$ boson, as well as, through the charged component of the extra doublets, with the $W$ boson.

As recently reviewed in [23] (see also e.g. [24,25]), DM interactions mediated exclusively by the Higgs and the $Z$ bosons are disfavored by DM Direct Detection (DD), expecially in the case that the DM is a dirac fermion [19,26,27].

In this work we will investigate in detail whether this problem can be encompassed by extending, with a second dou-

\footnotetext{
1 These kind of operators can be obtained by integrating out extra heavy states (see e.g. [16]).

2 An extended version of these setups has been recently proposed to account for neutrino masses as well [22].
} 
blet, the Higgs sector of the theory. A similar investigation has been already presented in [28], but with focus only on the possibility of a light pseudoscalar boson. While including this scenario in our discussion, we will, however, investigate the parameter space of the theory from a more general perspective. We will pinpoint, furthermore, the complementarity with constraints from searches at collider and in low energy processes of extra Higgs bosons.

The paper is organized as follows. We will first introduce, in Sect. 2, our model setup. Section 3 will be devoted to a brief review of the two-doublet extension of the Higgs sector and to the discussion of the theoretical and experimental limits which can impact the viable parameter space for DM. The most salient features of DM phenomenology will be then discussed in Sect. 4. We will finally present and discuss our findings in Sect. 5.

\section{The model}

\subsection{HDM and coupling to the SM}

We will adopt, for our study, a 2HDM model described by the the following potential:

$$
\begin{aligned}
V\left(H_{1}, H_{2}\right)= & m_{11}^{2} H_{1}^{\dagger} H_{1}+m_{22}^{2} H_{2}^{\dagger} H_{2} \\
& -m_{12}^{2}\left(H_{1}^{\dagger} H_{2}+\text { h.c. }\right)+\frac{\lambda_{1}}{2}\left(H_{1}^{\dagger} H_{1}\right)^{2} \\
& +\frac{\lambda_{2}}{2}\left(H_{2}^{\dagger} H_{2}\right)^{2} \\
& +\lambda_{3}\left(H_{1}^{\dagger} H_{1}\right)\left(H_{2}^{\dagger} H_{2}\right)+\lambda_{4}\left(H_{1}^{\dagger} H_{2}\right)\left(H_{2}^{\dagger} H_{1}\right) \\
& +\frac{\lambda_{5}}{2}\left[\left(H_{1}^{\dagger} H_{2}\right)^{2}+\text { h.c. }\right],
\end{aligned}
$$

where two doublets are defined by:

$H_{i}=\left(\begin{array}{c}\phi_{i}^{+} \\ \left(v_{i}+\rho_{i}+i \eta_{i}\right) / \sqrt{2}\end{array}\right), \quad i=1,2$,

further assuming that all the couplings above are real. Equation 1 further assumes the absence of two additional couplings $\lambda_{6}$ and $\lambda_{7}$. This is for example obtained by assuming that the Higgs doublets transform as $H_{1} \rightarrow H_{1}$ and $H_{2} \rightarrow-H_{2}$ under a discrete $\mathbb{Z}_{2}$ symmetry [29] (see also [30-32]). We also introduce, as usual, the $\beta$ angle defined as $v_{2} / v_{1}=\tan \beta$.

Imposing $\mathrm{CP}$-conservation in the scalar sector, the spectrum of physical states is constituted by two $\mathrm{CP}$ even neutral states, $h$, identified with the $125 \mathrm{GeV}$ Higgs, and $H$, the CP-odd Higgs $A$ and finally the charged Higgs $H^{ \pm}$. The transition from the interaction basis $\left(H_{1}, H_{2}\right)^{T}$ to the mass basis $\left(h, H, A, H^{ \pm}\right)$depends on two mixing angles, $\alpha$ and $\beta$ according the following relations:

$$
\begin{aligned}
& \left(\begin{array}{l}
\phi_{1}^{+} \\
\phi_{2}^{+}
\end{array}\right)=\left(\begin{array}{cc}
\cos \beta & -\sin \beta \\
\sin \beta & \cos \beta
\end{array}\right)\left(\begin{array}{l}
G^{+} \\
H^{+}
\end{array}\right), \\
& \left(\begin{array}{l}
\eta_{1} \\
\eta_{2}
\end{array}\right)=\left(\begin{array}{cc}
\cos \beta & -\sin \beta \\
\sin \beta & \cos \beta
\end{array}\right)\left(\begin{array}{c}
G^{0} \\
A
\end{array}\right) \\
& \left(\begin{array}{l}
\rho_{1} \\
\rho_{2}
\end{array}\right)=\left(\begin{array}{cc}
\cos \alpha & -\sin \alpha \\
\sin \alpha & \cos \alpha
\end{array}\right)\left(\begin{array}{l}
H \\
h
\end{array}\right)
\end{aligned}
$$

with $G^{0}, G^{+}$being Goldstone bosons, eaten, after EW symmetry breaking, as longitudinal degrees of freedom, by the SM gauge bosons. The quartic couplings of the scalar potential (1) can be expressed as function of the masses of the physical states as:

$$
\begin{aligned}
& \lambda_{1}=\frac{1}{v^{2}}\left[-\tan ^{2} \beta M^{2}+\frac{\sin ^{2} \alpha}{\cos ^{2} \beta} m_{h}^{2}+\frac{\cos ^{2} \alpha}{\cos ^{2} \beta} m_{H}^{2}\right], \\
& \lambda_{2}=\frac{1}{v^{2}}\left[-\frac{1}{\tan ^{2} \beta} M^{2}+\frac{\cos ^{2} \alpha}{\sin ^{2} \beta} m_{h}^{2}+\frac{\sin ^{2} \alpha}{\sin ^{2} \beta} m_{H}^{2}\right], \\
& \lambda_{3}=\frac{1}{v^{2}}\left[-M^{2}+2 m_{H^{ \pm}}^{2}+\frac{\sin 2 \alpha}{\sin 2 \beta}\left(m_{H}^{2}-m_{h}^{2}\right)\right], \\
& \lambda_{4}=\frac{1}{v^{2}}\left[M^{2}+m_{A}^{2}-2 m_{H^{ \pm}}^{2}\right], \\
& \lambda_{5}=\frac{1}{v^{2}}\left[M^{2}-m_{A}^{2}\right],
\end{aligned}
$$

where $M \equiv m_{12} /\left(s_{\beta} c_{\beta}\right)$.

The SM fermions cannot couple freely with both Higgs doublets since, otherwise, FCNCs would be induced at tree level. Four specific configurations, labelled Type-I, Type-II, Lepton specific and Flipped, avoid this eventuality. In the physical basis for the scalar sector the interaction Lagrangian between the Higgses and the SM fermions reads:

$$
\begin{aligned}
-\mathcal{L}_{\text {yuk }}^{S M}= & \sum_{f=u, d, l} \frac{m_{f}}{v}\left[\xi_{h}^{f} \bar{f} f h+\xi_{H}^{f} \bar{f} f H-i \xi_{A}^{f} \bar{f} \gamma_{5} f A\right] \\
& -\left[\frac{\sqrt{2}}{v} \bar{u}\left(m_{u} \xi_{A}^{u} P_{L}+m_{d} \xi_{A}^{d} P_{R}\right) d H^{+}\right. \\
& \left.+\frac{\sqrt{2}}{v} m_{l} \xi_{A}^{l} \overline{\nu_{L}} l_{R} H^{+}+\text {h.c. }\right]
\end{aligned}
$$

where $v=\sqrt{v_{1}^{2}+v_{2}^{2}}=246 \mathrm{GeV}$. The coefficients $\xi_{h, H, A}^{u, d, l}$ are, in general functions of $\alpha, \beta$, and depend on how the SM fermions are coupled with the $H_{1}, H_{2}$ doublets. Their values, for the four FCNC preserving 2HDM realizations, are summarized in Table 1.

Constraints from $125 \mathrm{GeV}$ Higgs signal strength limit the values of $\alpha$ and $\beta$. These bounds will be discussed in more detail below. We just mention that one can automatically comply with them by going to the so-called "alignment" limit, i.e. $\beta-\alpha=\frac{\pi}{2}$, which makes automatically the couplings of the $h$ state SM like, i.e. $\xi_{h}^{u, d, l}=1$, and the other $\xi$ 
Table 1 Couplings of the Higgses to the SM fermions as a function of the angles $\alpha$ and $\beta$ and in the alignment limit where $(\beta-\alpha) \rightarrow \pi / 2$

\begin{tabular}{|c|c|c|c|c|}
\hline & Type-I & Type-II & Lepton-specific & Flipped \\
\hline$\xi_{h}^{u}$ & $c_{\alpha} / s_{\beta} \rightarrow 1$ & $c_{\alpha} / s_{\beta} \rightarrow 1$ & $c_{\alpha} / s_{\beta} \rightarrow 1$ & $c_{\alpha} / s_{\beta} \rightarrow 1$ \\
\hline$\xi_{h}^{d}$ & $c_{\alpha} / s_{\beta} \rightarrow 1$ & $-s_{\alpha} / c_{\beta} \rightarrow 1$ & $c_{\alpha} / s_{\beta} \rightarrow 1$ & $-s_{\alpha} / c_{\beta} \rightarrow 1$ \\
\hline$\xi_{h}^{l}$ & $c_{\alpha} / s_{\beta} \rightarrow 1$ & $-s_{\alpha} / c_{\beta} \rightarrow 1$ & $-s_{\alpha} / c_{\beta} \rightarrow 1$ & $c_{\alpha} / s_{\beta} \rightarrow 1$ \\
\hline$\xi_{H}^{u}$ & $s_{\alpha} / s_{\beta} \rightarrow-t_{\beta}^{-1}$ & $s_{\alpha} / s_{\beta} \rightarrow-t_{\beta}^{-1}$ & $s_{\alpha} / s_{\beta} \rightarrow-t_{\beta}^{-1}$ & $s_{\alpha} / s_{\beta} \rightarrow-t_{\beta}^{-1}$ \\
\hline$\xi_{H}^{d}$ & $s_{\alpha} / s_{\beta} \rightarrow-t_{\beta}^{-1}$ & $c_{\alpha} / c_{\beta} \rightarrow t_{\beta}$ & $s_{\alpha} / s_{\beta} \rightarrow-t_{\beta}^{-1}$ & $c_{\alpha} / c_{\beta} \rightarrow t_{\beta}$ \\
\hline$\xi_{H}^{l}$ & $s_{\alpha} / s_{\beta} \rightarrow-t_{\beta}^{-1}$ & $c_{\alpha} / c_{\beta} \rightarrow t_{\beta}$ & $c_{\alpha} / c_{\beta} \rightarrow t_{\beta}$ & $s_{\alpha} / s_{\beta} \rightarrow-t_{\beta}^{-1}$ \\
\hline$\xi_{A}^{u}$ & $t_{\beta}^{-1}$ & $t_{\beta}^{-1}$ & $t_{\beta}^{-1}$ & $t_{\beta}^{-1}$ \\
\hline$\xi_{A}^{d}$ & $-t_{\beta}^{-1}$ & $t_{\beta}$ & $-t_{\beta}^{-1}$ & $t_{\beta}$ \\
\hline$\xi_{A}^{l}$ & $-t_{\beta}^{-1}$ & $t_{\beta}$ & $t_{\beta}$ & $-t_{\beta}^{-1}$ \\
\hline
\end{tabular}

parameters only dependent on $\tan \beta$. A further implication of the alignment limit is that the coupling of the $\mathrm{CP}$-even state $H$ with the $W$ and $Z$ bosons is null (the couplings of the $A$ boson with $Z Z$ and $W W$ are null as long $\mathrm{CP}$ is conserved in the Higgs sector). In our study we will keep as free as possible the parameters of the Higgs sector. We will then do not strictly impose the alignment limit but rather keep $\alpha$ and $\beta$ as free parameters and impose on them the relevant constraints.

\subsection{Coupling to the DM}

In the scenario under investigation, the DM arises from the mixture of a SM singlet $N^{\prime}$ and the neutral components of two (Weyl) $S U$ (2) doublets $L_{L}$ and $L_{R}$ with hypercharges equal, respectively, to -1 and +1 , defined as:

$L_{L}=\left(\begin{array}{c}N_{L} \\ E_{L}\end{array}\right), \quad L_{R}=\left(\begin{array}{c}-E_{R} \\ N_{R}\end{array}\right)$

The new fermions are coupled with two Higgs doublets $H_{1}$ and $\mathrm{H}_{2}$ according the following Lagrangian:

$$
\begin{aligned}
\mathcal{L}= & -\frac{1}{2} M_{N} N^{\prime 2}-M_{L} L_{L} L_{R}-y_{i}^{L} L_{L} H_{i} N^{\prime} \\
& -y_{i}^{R} \bar{N}^{\prime} \tilde{H}_{i}^{\dagger} L_{R}+\text { h.c. },
\end{aligned}
$$

$i=1,2$

Notice that, given their quantum numbers, the new fermions could be coupled, through the Higgs, also with SM leptons and, hence, mix with them after EW symmetry breaking. This would imply, in particular, that the DM is not stable. To avoid this possibility we assume the existence of a discrete $\mathbb{Z}_{2}$ symmetry, which we label as $\mathbb{Z}_{2}^{\mathrm{DM}}$, under which the new and the SM fermions are, respectively, odd and even.

Similarly to SM fermions, it is appropriate to avoid to couple these new states freely with both Higgs doublets. Several different configurations are nevertheless still possible (see for example [28]). For definiteness we will then focus, along this work, on two specific configurations (we will comment on other possibilities in Appendix B): $y_{1}^{L}=y_{1}, y_{2}^{L}=y_{2}^{R}=0$, $y_{1}^{R}=y_{2}$ and $: y_{1}^{L}=y_{1}, y_{2}^{L}=y_{1}^{R}=0, y_{2}^{R}=y_{2}$. For the first configuration we will further assume that the SM fermions are coupled as in the standard Type-I 2HDM and globally label as "Type-I" the model defined in this way. The second configuration for the new fermions is, instead, accompanied by coupling of the SM fermions with $H_{1}, H_{2}$ as in the Type-II 2HDM and we will refer this scenario as "Type-II' model.

After EW symmetry breaking, mixing between $N^{\prime}$ and the neutral components of $L_{L}$ and $L_{R}$ occurs, so that the physical spectrum of the new fermions is represented by three Majorana fermions $\psi_{i=1,3}$ defined by:

$\psi_{i}=N^{\prime} U_{i 1}+N_{L} U_{i 2}+N_{R} U_{i 3}$,

and one charged dirac fermion $\psi_{ \pm}$with mass $m_{\psi_{ \pm}} \approx M_{L}$. The matrix $U$ diagonalizes a mass matrix of the form:

$M=\left(\begin{array}{ccc}M_{N} & \frac{y_{1} v_{1}}{\sqrt{2}} & \frac{y_{2} v_{2}}{\sqrt{2}} \\ \frac{y_{1} v_{1}}{\sqrt{2}} & 0 & M_{L} \\ \frac{y_{2} v_{2}}{\sqrt{2}} & M_{L} & 0\end{array}\right)$,

where, for definiteness we have considered the Type-II model.

Similarly to [18,20,28], we will adopt, in spite of $y_{1}, y_{2}$, the free parameters $y, \theta$ defined by:

$y_{1}=y \cos \theta \quad y_{2}=y \sin \theta$

In the mass basis, the relevant interaction Lagrangian for DM phenomenology reads: 


$$
\begin{aligned}
\mathcal{L}= & \overline{\psi^{-}} \gamma^{\mu}\left(g_{W \chi_{i}}^{V}-g_{W \chi_{i}}^{A} \gamma_{5}\right) \psi_{i} W_{\mu}^{-}+\text {h.c. } \\
& +\frac{1}{2} \sum_{i, j=1}^{3} \overline{\psi_{i}} \gamma^{\mu}\left(g_{Z \psi_{i} \psi_{j}}^{V}-g_{Z \psi_{i} \psi_{j}}^{A} \gamma_{5}\right) Z_{\mu} \psi_{j} \\
& +\frac{1}{2} \sum_{i, j=1}^{3} \overline{\psi_{i}}\left(y_{h \psi_{i} \psi_{j}} h+y_{H \psi_{i} \psi_{j}} H+y_{A \psi_{i} \psi_{j}} \gamma_{5} A\right) \psi_{j} \\
& + \text { h.c } \\
& +\overline{\psi^{-}}\left(g_{H^{ \pm} \psi_{i}}^{S}-g_{H^{ \pm} \psi_{i}}^{P} \gamma_{5}\right) \psi_{i} H^{-}+\text {h.c. } \\
& -e A_{\mu} \overline{\psi^{-}} \gamma^{\mu} \psi^{-} \\
& -\frac{g}{2 \cos \theta_{W}}\left(1-2 \sin ^{2} \theta_{W}\right) Z_{\mu} \overline{\psi^{-}} \gamma^{\mu} \psi^{-}+\text {h.c. }
\end{aligned}
$$

where $\theta_{W}$ is the Weinberg's angle, $A_{\mu}$ and $Z_{\mu}$ are, respectively, the photon and $Z$ fields, and with:

$$
\begin{aligned}
g_{W \chi_{i}}^{V}= & \frac{g}{2 \sqrt{2}}\left(U_{i 3}-U_{i 2}^{*}\right) \\
g_{W \chi_{i}}^{A}= & -\frac{g}{2 \sqrt{2}}\left(U_{i 3}+U_{i 2}^{*}\right) \\
g_{Z \psi_{i} \psi_{j}}^{V}= & \frac{g}{4 \cos \theta_{W}}\left[\left(U_{i 3} U_{j 3}^{*}-U_{i 2} U_{j 2}^{*}\right)\right. \\
& \left.-\left(U_{i 3}^{*} U_{j 3}-U_{i 2}^{*} U_{j 2}\right)\right] \\
g_{Z \psi_{i} \psi_{j}}^{A}= & \frac{g}{4 \cos \theta_{W}}\left[\left(U_{i 3} U_{j 3}^{*}-U_{i 2} U_{j 2}^{*}\right)\right. \\
& \left.+\left(U_{i 3}^{*} U_{j 3}-U_{i 2}^{*} U_{j 2}\right)\right] \\
g_{h \psi_{i} \psi_{j}=}= & \frac{1}{2 \sqrt{2}}\left[U_{i 1}\left(y_{1} N_{1}^{h} U_{i 2}+y_{2} N_{2}^{h} U_{i 3}\right)+(i \leftrightarrow j)\right] \\
g_{H \psi_{i} \psi_{j}=}= & \frac{1}{2 \sqrt{2}}\left[U_{i 1}\left(y_{1} N_{1}^{H} U_{i 2}+y_{2} N_{2}^{H} U_{i 3}\right)+(i \leftrightarrow j)\right] \\
g_{A \psi_{i} \psi_{j}=}= & -\frac{i}{2 \sqrt{2}}\left[U_{i 1}\left(y_{1} N_{1}^{A} U_{i 2}+y_{2} N_{2}^{A} U_{i 3}\right)+(i \leftrightarrow j)\right] \\
g_{H^{ \pm} \psi_{i}}^{S}= & \frac{1}{2} U_{i 1}\left(y_{1} N_{1}^{H^{ \pm}}+y_{2} N_{2}^{H^{ \pm}}\right) \\
g_{H^{ \pm} \psi_{i}}^{P}= & \frac{1}{2} U_{i 1}\left(y_{1} N_{1}^{H^{ \pm}}-y_{2} N_{2}^{H^{ \pm}}\right)
\end{aligned}
$$

where:

$N_{1}^{h}=N_{2}^{h}=-\sin \alpha, \quad N_{1}^{H}=N_{2}^{H}=\cos \alpha$,

$$
N_{1}^{A}=N_{2}^{A}=-\sin \beta \text {, }
$$

$N_{1}^{H^{ \pm}}=N_{2}^{H^{ \pm}}=-\sin \beta \quad$ (Type-I)

$N_{1}^{h}=-\sin \alpha, \quad N_{2}^{h}=\cos \alpha, \quad N_{1}^{H}=\cos \alpha, \quad N_{2}^{H}=\sin \alpha$

$N_{1}^{A}=-\sin \beta \quad N_{2}^{A}=\cos \beta$,

$N_{1}^{H^{ \pm}}=-\sin \beta, \quad N_{2}^{H^{ \pm}}=\cos \beta \quad$ (Type-II)

Notice, in particular, that, as expected from its Majorana nature, the vectorial coupling of the DM with the $Z$ boson is null.

\section{Constraints on the Higgs sector}

3.1 Bounds on the potential

The quartic couplings $\lambda_{i=1,5}$ should comply with a series of constraints coming from the unitarity and boundness from below of the scalar potential as well as perturbativity (see for example. [33,34] for more detailed discussions). These bounds, can be translated, through Eq. 4, into bounds on the masses of the new Higgs bosons as function of the angles $\alpha$ and $\beta$.

For completeness we list below the main constraints:

- Scalar potential bounded from below:

$\lambda_{1,2}>0, \lambda_{3}>-\sqrt{\lambda_{1} \lambda_{2}}$, and $\lambda_{3}+\lambda_{4}-\left|\lambda_{5}\right|>-\sqrt{\lambda_{1} \lambda_{2}}$;

- tree level s-wave unitarity:

$$
\left|a_{ \pm}\right|,\left|b_{ \pm}\right|,\left|c_{ \pm}\right|,\left|f_{ \pm}\right|,\left|e_{1,2}\right|,\left|f_{1}\right|,\left|p_{1}\right|<8 \pi
$$

where:

$$
\begin{aligned}
a_{ \pm} & =\frac{3}{2}\left(\lambda_{1}+\lambda_{2}\right) \pm \sqrt{\frac{9}{4}\left(\lambda_{1}-\lambda_{2}\right)^{2}+\left(2 \lambda_{3}+\lambda_{4}\right)^{2}} \\
b_{ \pm} & =\frac{1}{2}\left(\lambda_{1}+\lambda_{2}\right) \pm \sqrt{\left(\lambda_{1}-\lambda_{2}\right)^{2}+4 \lambda_{4}^{2}} \\
c_{ \pm} & =\frac{1}{2}\left(\lambda_{1}+\lambda_{2}\right) \pm \sqrt{\left(\lambda_{1}-\lambda_{2}\right)^{2}+4 \lambda_{5}^{2}} \\
e_{1} & =\lambda_{3}+2 \lambda_{4}-3 \lambda_{5}, \quad e_{2}=\lambda_{3}-\lambda_{5} \\
f_{+} & =\lambda_{3}+2 \lambda_{4}+3 \lambda_{5}, \quad f_{-}=\lambda_{3}+\lambda_{5} \\
f_{1} & =\lambda_{3}+\lambda_{4}, \quad p_{1}=\lambda_{3}-\lambda_{4}
\end{aligned}
$$

- global minimum in $\left(v_{1}, v_{2}\right)$ for the scalar potential [35]:

$$
m_{12}^{2}\left(m_{11}^{2}-m_{22}^{2} \sqrt{\lambda_{1} / \lambda_{2}}\right)\left(\tan \beta-\sqrt[4]{\lambda_{1} / \lambda_{2}}\right)>0
$$

where the mass parameters $m_{11}, m_{22}, m_{12}$ should satisfy:

$$
\begin{aligned}
m_{11}^{2} & +\frac{\lambda_{1} v^{2} \cos ^{2} \beta}{2}+\frac{\lambda_{3} v^{2} \sin ^{2} \beta}{2} \\
= & \tan \beta\left[m_{12}^{2}-\left(\lambda_{4}+\lambda_{5}\right) \frac{v^{2} \sin 2 \beta}{4}\right] \\
m_{22}^{2} & +\frac{\lambda_{2} v^{2} \sin ^{2} \beta}{2}+\frac{\lambda_{3} v^{2} \cos ^{2} \beta}{2} \\
= & \frac{1}{\tan \beta}\left[m_{12}^{2}-\left(\lambda_{4}+\lambda_{5}\right) \frac{v^{2} \sin 2 \beta}{4}\right] .
\end{aligned}
$$




\subsection{EWPT}

The presence of extra Higgs bosons affects the values of the Electroweak Precision Observables (EWPO), possibly making them to deviate from the SM expectations. One option to alleviate these tensions would be represented by imposing specific relations for the masses of the new states; for example deviations of the $T$ parameter can be avoided imposing a custodial symmetry [36,37]. As already pointed, for our DM analysis, we will nevertheless try to keep the parameters of the Higgs sector as free as possible. To identify the viable paramter space we have then computed the $S, T, U$ parameters using the functions provided, for example in [38-41], as function of the masses $m_{H}, m_{A}, m_{H^{ \pm}}$of the new Higgs bosons as well as function of $\alpha$ and $\beta$ (we remind we are not imposing the alignment limit) and determining the excluded model configurations through the following $\chi^{2}$ [42-44]:

$\chi^{2}=\sum_{i, j}\left(X_{i}-X_{i}^{\mathrm{SM}}\right)\left(\sigma_{i} V_{i j} \sigma_{j}\right)^{-1}\left(X_{j}-X_{j}^{\mathrm{SM}}\right)$

with $X=(S, T, U)$ while $X^{\mathrm{SM}}, \sigma$ and $V$ represent, respectively, the SM expectations of the Electroweak Precision Observables, the corresponding Standard deviation and covariant matrix. Their updated values have been provided here [45] and are also reported here for convenience:

$X^{\mathrm{SM}}=(0.05,0.09,0.01), \quad \sigma=(0.11,0.13,0.11)$

$V=\left(\begin{array}{lll}1 & 0.9 & -0.59 \\ 0.9 & 1 & -0.83 \\ -0.59 & -0.83 & 1\end{array}\right)$

We have imposed to each model point to do not induce a deviation, for the EWPO, beyond $3 \sigma$ from their best fit values.

The EWPO can be as well affected by the fermionic sector of the theory $[39,40,46-48]$. The corresponding contributions to the STU parameters depend on masses and on the Yukawa couplings for the new fermions. In particular in the limit in which the latter parameters vanish also the contributions to EWPO from the new fermions become negligible.

On general grounds one should include in Eq. 23 also the contribution from the new fermionic sector to the EWPO. However, in agreement with the findings of [20] (for the computation of the EWPO we have adapted the expressions provided in [48]), the new fermionic sector mostly affects the $\mathrm{T}$ parameter. Given this, we have imposed a priori that the deviation $\Delta T$ of the $T$ parameter induced by the new fermions is neglible and then used Eq. 23 to constrain the parameters of the scalar sector. ${ }^{3}$

\footnotetext{
3 One could have considered, as done for example in [27], the possibility of cancellations between the contributions to the EWPO from the new
}

The deviation $\Delta T$ induced by the DM sector scales with the combination $\left(y_{1}^{2} v_{a}^{2}-y_{2}^{2} v_{b}^{2}\right)^{2}$, with $a, b$ set appropriately to 1,2 , depending on whether the Type-I or the Type-II models are considered, and then vanishes in the limit $\tan \theta \rightarrow 1$ ( $\tan \beta \rightarrow 1$ would be also required for the Type-II model). We have verified that $\Delta T$ remains sensitively below the experimental exclusion unless $y>1$ and $|\tan \theta|>10$ (in the case of the Type-II model the contribution to the $T$ parameter increases also with $\tan \beta$. As will be clarified below, moderate-high values of $\tan \beta$ are also disfavoured by LHC constraints as well as by DM phenomenology and, hence, have not been considered). As already mentioned, for the study of the DM phenomenology we have chosen assignations of the parameters, in the new fermionic sector, automatically complying with the bounds from EWPT.

\subsection{Collider searches of the new Higgs bosons}

$H$ and $A$ bosons can be resonantly produced at colliders through gluon fusion, ${ }^{4}$ and are, hence, object of searches in a broad variety of final states ranging from $\tau^{+} \tau^{-}[49,50] \bar{b} b$ [51-54], $\bar{t} t$ [55], diboson [56-63], $h h$ (only for $H$ ) [64-69], $Z h$ (only for $A$ ) [70-72], $A \rightarrow Z H$ and $H \rightarrow Z A[73,74]$.

Among these, the most effective bounds come from searches of $\tau^{+} \tau^{-}$pairs, which exclude, for the Type-II model, moderate-high values of $\tan \beta$, above 10. Searches of diboson final states and of the process $A \rightarrow Z h(H)$ (as well as the process with inverse mass ordering) can, in addition, constrain deviations from the alignment limit.

The strongest limits for the latter come, however, from the Higgs signal strenght. For our analysis we have adopted the limit determined in [27] combining the constraints on the Higgs signal strenght into $\gamma \gamma, W^{+} W^{-}, Z Z, \bar{b} b$ and $\tau^{+} \tau^{-}$ from the LHC run I $[75,76]$ (see also [77]). ${ }^{5}$ The excluded regions in the bidimensional plane $(\cos (\beta-\alpha), \tan \beta)$ are sketched in Fig. 1. As evident sizable deviations from the alignment limit are allowed in the case of the Type-I 2HDM. This is not the case, on the contrary, for the Type-II 2HDM, ad exception of a very narrow window corresponding to the so called "wrong-sign" Yukawa regime [81-83], i.e. the case in which the couplings of the state $h$ with the down type

(Footnote 3 continued)

scalar and fermionic sectors. This would allow for higher values of the yukawa couplings, provided very strong constraints in the assignation of $m_{A}, m_{H}, m_{H^{ \pm}}$. We have not considered this scenario here.

${ }^{4}$ For 2 HDM realizations with $\xi_{H_{A}}^{d}$ enhanced with $\tan \beta$ also $\bar{b} b$ fusion can play a relevant role [27]. The Type-II model considered in this work features this property. However, as discussed in the text, limits from searches of resonances decaying into $\tau^{+} \tau^{-}$final states put strong limits on the size of $\tan \beta$ so that one can realiably assume gluon fusion as the most relevant production process.

5 Further stronger constraints would be obtained by considering Run II data [78-80]. These have not a relevant impact in our analysis. 

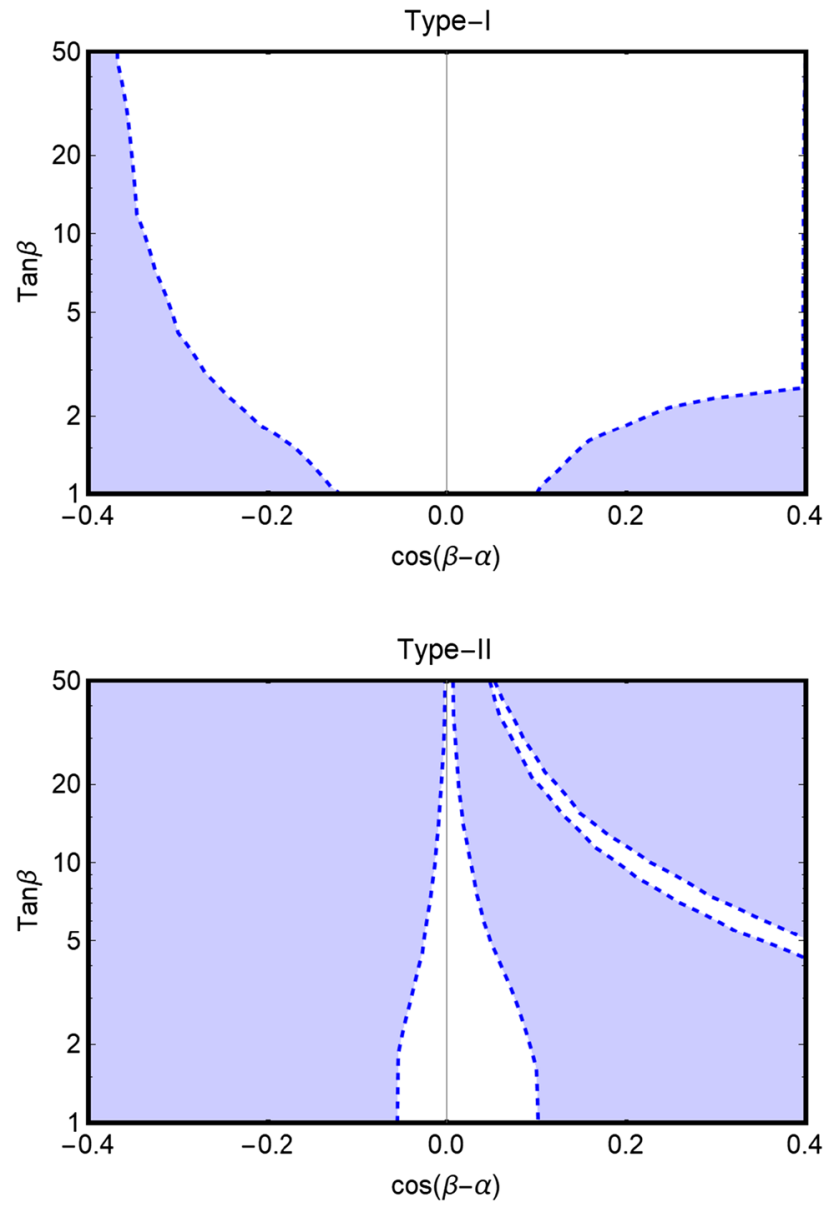

Fig. 1 Limits on deviations (the white regions are allowed) from the alignment limit in the for Type-I (upper panel) and Type-II (bottom panel) $2 \mathrm{HDM}$

quarks are opposite in sign but equal in absolute values with respect to their SM corresponding.

Similarly to [28], we will include in our analysis also the case in which $m_{A}<m_{h}$. In such a case one should take into account possible limits on the process $h \rightarrow A A$. This can be generically constrained through the Higgs signal strenght (i.e. one generically imposes that the branching fraction of this decay does not exceed the allowed value of the invisible branching fraction of the Higgs. This bound is effective in the whole range of masses for which the decay $h \rightarrow A A$ is kinematically allowed) as well as through dedicated searches [84] (limits are effective only for some range of masses). More contrived are instead the prospects for signals not related to Higgs decays, see anyway $[85,86]$.

Concerning the charged Higgs boson we have first of all a limit $m_{H^{ \pm}} \gtrsim 80 \mathrm{GeV}$ from LEP [87]. Moving to LHC constraints, these come, for $m_{H^{ \pm}}<m_{t}$, from searches of top decays $t \rightarrow H^{ \pm} b$ with $H^{ \pm}$[88-91] decaying into $\tau v_{\tau}$ or $c s$. The corresponding limits have been reformulated in [92] for different realizations of the 2HDM. Values of the masses of the charged Higgs for which the decay is kinematically allowed are excluded for $\tan \beta \lesssim 10$ in the Type-I 2HDM and irrespective of the value of $\tan \beta$ for the Type-II scenario. For $m_{H^{ \pm}}>m_{t}$ searches rely on direct production of the charged Higgs in association with a top and a bottom quark, followed by the decay of the former in $\tau v$ [90,9395] or $t b$ [94]. Associated limits are not competitive with the others discussed in here and will be then neglected. The charged Higgs feels, indirectly, also limits from searches of the neutral Higgs bosons since the conditions on the quartic couplings 18-19 impose relations between the masses of the new Higgs bosons (see e.g [92]).

\subsection{Limits from flavour}

While it is possible to avoid that the couplings of the extra Higgs bosons with SM fermions induce FCNC at the tree level, they can impact flavour violating transitions at the loop level. The strongest limits come from processes associated to $b \rightarrow s$ transitions. Their rates are mostly sensitive to $m_{H^{ \pm}}$ and $\tan \beta$. Experimental limits are formulated in terms of these parameters. The most stringent come from the $B \rightarrow$ $X_{s} \gamma$ process [96] and are particularly severe in the case of Type-II model, excluding $m_{H^{ \pm}}<570 \mathrm{GeV}$ irrespective of $\tan \beta$ [97]. Much better is, instead, the situation of the TypeI model where we have just the approximate lower bound $\tan \beta \gtrsim 2$. A similar exclusion, for both Type-I and Type-II models is also provided by the processes $B_{S} \rightarrow \mu^{+} \mu^{-}$and $B \rightarrow K \mu^{+} \mu^{-}$[43].

\subsection{Scanning the parameter space}

The bounds discussed in the previous subsection have been applied to a parameter scan over the following ranges ${ }^{6}$

$$
\begin{aligned}
& \tan \beta \in[1,50] \quad \alpha \in\left[-\frac{\pi}{2}, \frac{\pi}{2}\right] \\
& m_{H}=M \in\left[m_{h}, 1 \mathrm{TeV}\right], \quad m_{H^{ \pm}} \in\left[m_{W}, 1 \mathrm{TeV}\right] \\
& m_{A} \in[20 \mathrm{GeV}, 1 \mathrm{TeV}],
\end{aligned}
$$

As already said, we will include in our analysis a very light pseudoscalar $A$. For this reason we have considered a minimal value of $20 \mathrm{GeV}$ for its mass in the scan. More precisely the scan consisted into random extracting, with flat priors, the model parameters within the ranges reported and keeping the sets of model points satisfying the following constraints:

\footnotetext{
${ }^{6}$ For simplicity we have limited to 50 the maximal value of $\tan \beta$ in our scan of the parameter space. This is easily understood in the case of the Type-II model since already moderate values of $\tan \beta$ are expected to be excluded by LHC searches of $A \rightarrow \tau \tau$. In the case of the Type-I model very high values of $\tan \beta$ would correspond to a "fermiophobic" limit for the interactions of the new Higgs bosons, see e.g. [27] which is disfavoured by DM phenomenology (see next section).
} 
- the constraints $18-21$ on the quartic couplings $\lambda_{1,5}$ determined, according 4 , as function of $m_{H}, m_{A}, m_{H^{ \pm}}, \alpha, \beta$;

- the bounds from Higgs signal strengths, imposing that the values of $\alpha, \beta$ for each model point lie in the white regions of 1 ;

- bounds from EWPT, according the procedure depicted in sec. III B, and the flavour constraints, illustrated in sec. III D.

- compatibility with null results from searches of new Higgs bosons at the LHC. To this purpose we have determined the production cross-sections of the Higgs bosons through the same procedure already employed in [27]. We have then adopted the cross-section provided by the LHC Higgs Cross Section Working Group [98], which have been determined through the package SusHi [99]. In the case of the Type-II model it has been convenient, to our purposes, to adopt the cross-sections computed for the hMSSM [100,101], given the similarity of setups. For what concerns the Type-I model, as discussed in [27], given the fact that the gluon fusion is the dominant production channel and that all the couplings of the H/A bosons with SM fermions have the same dependence on $\tan \beta$, the production cross sections could be simply computed by rescaling by a $1 / \tan ^{2} \beta$ factor the hMSSM cross sections computed for $\tan \beta=1$.

This procedure has been performed for both Type-I and TypeII 2HDM individually. For each model a sample of 10,0000 viable solutions has been collected.

Among the viable model points we have identified some benchmark assignations for $m_{H}, m_{A}, m_{H^{ \pm}}, \alpha, \beta$ which will be used for the DM analysis. We have investigated, in particular, the possibility of obtaining a light mass of the pseudoscalar $A$, possibly lighter than the SM like Higgs $h$, while keeping the masses of the other Higgs states above few hundreds GeV. This possibility is easily realized in the Type-I model provided that $m_{A} \gtrsim 60 \mathrm{GeV}$, in order to kinematically forbid the $h \rightarrow A A$ decay. On the contrary, no viable model points, with a light pseudoscalar, have been found in the Type-II model. This is due to the strong bound on the mass of the charged Higgs from $b \rightarrow s$ transitions (see Sec. III D) in combination with the strong limits on $\cos (\beta-\alpha)$ from the Higgs signal strengths, which disfavour a sizeable hierarchy between the masses of the different Higgs states. The combination of these two constraints limited the range of viable pseudoscalar masses to $m_{A} \gtrsim 500 \mathrm{GeV}^{7}$

\footnotetext{
7 As evidenced by the right panel of Fig. 1, and already discussed in the text, a sizeable deviation from the alignment limit in the Type-II model is actually still allowed in a narrow strip for $\cos (\beta-\alpha)>0$ region, dubbed wrong sign Yukawa region. In this region the hierarchy between the mass of the pseudoscalar and the charged Higgs can be augmented. We have found that in this region the lower bound on $m_{A}$,
}

We then illustrate in the next section the main constraints coming from DM phenomenology.

\section{DM constraints}

\subsection{Relic density}

According to the WIMP paradigm, the DM has sizable enough interactions with the SM particles to be in thermal equilibrium in the Early stages of the history of the Universe. At a later stage the interaction rate of the DM fell below the Hubble expansion rate causing the freeze-out of the DM at temperatures of the order of $\frac{1}{20}-\frac{1}{30}$ the DM mass. Assuming standard cosmological history, the DM relic density, $\Omega_{\psi_{1}} h^{2}$, is determined by a single particle physics input, i.e. the DM thermally averaged pair annihilation cross-section. The relation between these two quantities is given by [102]:

$\Omega_{\psi_{1}} h^{2} \approx 8.76 \times 10^{-11} \mathrm{GeV}^{-2}\left[\int_{T_{0}}^{T_{f}} g_{*}^{1 / 2}\left\langle\sigma_{\mathrm{eff}} v\right\rangle \frac{d T}{m_{\psi_{1}}}\right]^{-1}$

where $T_{0}$ and $T_{f}$ represent, respectively, the present time and freeze-out temperatures while $g_{*}^{1 / 2}$ is a function of the relavistic degrees of freedom at the temperature $T$ [102]. $\left\langle\sigma_{\text {eff }} v\right\rangle$ is the effective annihilation cross-section [103]:

$\left\langle\sigma_{\mathrm{eff}} v\right\rangle=\sum_{i, j \in \psi_{1,2,3}, \psi^{ \pm}}\left\langle\sigma_{i j} v_{i j}\right\rangle \frac{n_{i}}{n_{i, e q}} \frac{n_{j}}{n_{j, e q}}$

including coannihilation effects from the additional neutral and charged states belonging to the Dark Matter sector. Coannihilation effects are expected to be important in the case $M_{L} \lesssim M_{N}$, corresponding to a DM with sizeable or even dominant doublet component, implying that at least the charged fermion $\psi^{ \pm}$is very close in mass to it.

We remind that the precise experimental determination $\Omega h^{2} \approx 0.12$ [1] is matched by a value of $\left\langle\sigma_{\text {eff }} v\right\rangle$ of the order of $10^{-26} \mathrm{~cm}^{3} \mathrm{~s}^{-1}$.

In the model considered in this work, a huge variety of processes contributes to $\left\langle\sigma_{\text {eff }} v\right\rangle$. For what regards DM pair annihilations, the most commonly considered are the annihilation into SM fermion pairs, $W W, Z Z$ and $Z h$, originated by s-channel exchange of the $h, H, A$ bosons as well as, in the case of annihilation into gauge boson pairs, tchannel exchange of the DM and the other new fermions. In this work we will put particular attention also to the case

(Footnote 7 continued) indirectly induced by the one on $m_{H^{ \pm}}$from $\operatorname{Br}\left(B \rightarrow X_{s} \gamma\right)$, reduces to $m_{A} \gtrsim 300 \mathrm{GeV}$. 

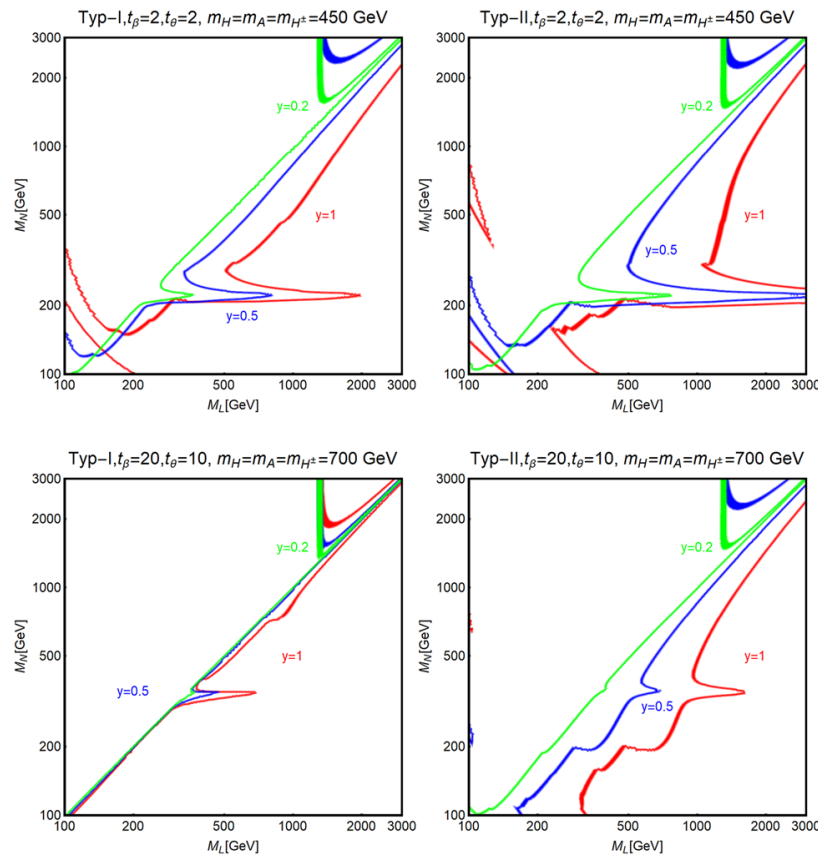

Fig. 2 Isocontours of the correct DM relic density in the bidimensional plane $\left(M_{N}, M_{L}\right)$ for Type-I 2HDM (abbreviated as Typ-I in the plot) (upper panel) and Type-II (abbreviated at Typ-II) models (lower panel), for three values of $y$, namely $0.2,0.5$ and 1 , and some assignations of the other relevant parameters, reported on top of the single panels

in which some of new Higgs bosons, in particular the pseudoscalar $A$, are lighter than the DM. This allows for additional annihilation channels into higgs bosons pairs, namely $H H$, $h A H A, A A, H^{+} H^{-}$, as well as gauge-higgs bosons final states as, $W^{ \pm} H^{\mp}, Z H$ and $Z A$. As already mentioned, this broad collection of processes is further enriched by coannihilations, i.e. annihilation processes with one or both DM initial states are replaced by the other fermions $\psi_{2,3}$ and $\psi^{ \pm}$.

All the possible DM annihilation channels have been included in our numerical study, performed through the package micrOMEGAs [104]. The DM relic density has been numerically determined with great accuracy by computing all the relevant annihilation processes, including coannihilations, adopting the Feynman rules, correspoding to the model under investigation, determined by the package Feynrules [105]. For a better insight, we report, nevertheless, in appendix A, the expressions of some phenomenologically interesting channels, by making use of the velocity expansion, $\langle\sigma v\rangle=a+b v_{\psi}^{2}$.

We have reported in Fig. 2 the isocontours corresponding to the correct DM relic density, $\Omega h^{2} \approx 0.12$ [1], in the bidimensional plane $\left(M_{L}, M_{N}\right)$ and for some assignations of the parameter of the model. For simplicity we have assumed $m_{H}=m_{A}=m_{H^{ \pm}}$and the alignment limit for the couplings of the Higgs bosons with SM states. In each panel of the figure we have considered three assignations of $y$, namely $0.2,{ }^{8} 0.5$ and 1 .

The shape of the isocontours can be understood as follows. The couplings of the DM, summarized by 16, depend on the size of the Yukawa couplings $y_{1}$ and $y_{2}$, parametrized by $y$ and $\tan \theta$, and on the elements of the mixing matrix $U$. Being $y$ and $\tan \theta$ fixed, as well as the masses of the Higgs bosons and $\tan \beta$, hence setting the coupling of the DM with the SM fermions, the DM annihilation cross-section is basically determined by the elements of $U$ according the values of $M_{N}$ and $M_{L}$. For $M_{L}<M_{N}$ the DM is mostly doublet like. Its relic density is essentially determined by (co)annihilations into gauge bosons. Being the size of the interactions set by the gauge couplings the DM relic density depends essentially on the DM mass. The experimentally favoured value is achieved for DM mass of the order of $1.1 \mathrm{TeV}$, lower (higher) values of the mass correspond to under(over)abundant DM. In agreement with this discussion the correct relic density isocontour is represented by a vertical line in the portion of the bidimensional plane for which $M_{L}$ is sensitively lower than $M_{N}$. In the case in which $M_{N}<M_{L}$ the DM becomes singlet like and its interactions are suppressed by the size of the element of $U$. In the regime $M_{N} \ll M_{L}$ the DM is overabundant ad exception of the "pole" regions, corresponding to the enhancement of the DM annihilation cross-section through schannel resonance, $m_{\psi_{1}}=\frac{m_{H, A}}{2}$ appearing as cuspy regions in Fig. 2. Far from the "poles" and the pure doublet regime, the isocontours of the correct relic density are determined by the size of the couplings of the DM with the Higgs bosons. For $y=1$ the correct relic density can be obtained for a singlet-like DM thanks, only in the Type-II model, to the $\tan \beta$ enhancement of the annihilation channels into $\bar{b} b$ and $\tau^{+} \tau^{-}$, and, when kinematically accessible, to the annihilation channels with new Higgses in the final state. Once the value of $y$ decreases, the isocontours tend to move towards the "well tempered" $[106,107]$ regime $M_{N} \sim M_{L}$. We notice in particular that, for $y=0.2$, the lines of the correct relic density, for all the four benchmarks, lie almost exactly in the $M_{N} \sim M_{L}$ diagonal.

A similar outcome would be also expected for lower values of $y$. For this reason we can focus, without loss of generality, to values $y \geq 0.2$. As a remark we notice that the TypeII benchmark reported in the top right panel of Fig. 2 is in tension with the constraints on the extensions of the Higgs sector discussed in the previous section. It should be then interpreted just as illustration.

\footnotetext{
8 With this assignation the coupling between the new SU(2) singlet and doublet fermions would be of the same size as the coupling of the Bino and the Higgsinos in the MSSM.
} 


\subsection{Direct detection}

In the considered scenario, the DM features both spin independent (SI) and spin dependent (SD) interactions with nuclei. The former are induced, at three level, by t-channel exchange of the CP-even $h$ and $H$ states. The corresponding cross-section reads (for definiteness we report the case of scattering on protons):

$\sigma_{\chi p}^{\mathrm{SI}}=\frac{\mu_{\chi}^{2}}{\pi} \frac{m_{p}^{2}}{v^{2}}\left|\sum_{q} f_{q}\left(\frac{g_{h \psi_{1} \psi_{1}} \xi_{h}^{q}}{m_{h}^{2}}+\frac{g_{H \psi_{1} \psi_{1}} \xi_{H}^{q}}{m_{H}^{2}}\right)\right|^{2}$

where $f_{q}^{p}$ are nucleon form factors (notice that the form factors corresponding to heavy quark are expressed in terms of the gluon form factor $[108,109]$ as $f_{c}^{p}=f_{c}^{p}=f_{t}^{p}=$ $\left.\frac{2}{27} f_{T G}\right)$

The strongest limits on spin independent interactions come, at the moment, from the XENON1T experiment [110] (slightly weaker limits have been provided, as well, by the LUX [111] and PANDAX [112]). In our analysis we will consider, in addition, the sensitivity prospectes of the LZ [113], XENONnT [114] (given their similarity, we will represent the sensitivity prospects of these experiments as a single line) and DARWIN [115].

Spin dependent interactions are originated, instead, by interactions of the DM with the $Z$ boson, giving the following cross-section:

$\sigma_{\chi p}^{\mathrm{SD}}=\frac{3 \mu_{\chi p}^{2}}{\pi m_{Z}^{4}}\left|g_{Z \psi_{1} \psi_{1}}^{A}\right|^{2}\left[g_{u}^{A} \Delta_{u}^{p}+g_{d}^{A}\left(\Delta_{d}^{p}+\Delta_{s}^{p}\right)\right]^{2}$

where $\Delta_{u, d, s}^{p}$ are again suitable structure functions ${ }^{9}$ while $g_{u}^{A}$ and $g_{d}^{A}$ represent the axial coupling of the $Z$ boson with, respectively, up-type and down-type quarks.

For our phenomenological analysis we have adopted the most recent limits provided by the LUX collaboration [116] (slightly weaker limits have been provided by PANDAX.)

As well known, Direct Detection constraints are mostly associated to SI interactions, because of the coherent enhanchment $\left(A^{2}\right)$ occuring when these are evaluated at the nuclear, rather than nucleon, level. However the different couplings of the DM with two mediators, the $h$ and $H$, can induce so called "blind spots" $[28,117-119]$, due to a possible destructive interference between diagrams with $h$ and $H$ exchange or to cancellations of the couplings of the DM with one or both the CP-even Higgs states. An analytical insight can be obtained by inspecting expressions of the couplings of the DM with the $h, H$ states as function of the DM mass

\footnotetext{
${ }^{9}$ For the numerical values of all structure functions we have adopted the default assignations of the micrOMEGAs package.
}

and of the $\left(M_{N}, M_{L}, y, \theta, \beta\right)$ parameters [28] (for simplicity we consider the alignment limit). These are for the Type-I model:

$g_{h \psi_{1} \psi_{1}}=y^{2} v \cos ^{2} \beta \frac{m_{\psi_{1}}+M_{L} \sin 2 \theta}{2 M_{L}^{2}+4 M_{N} m_{\psi_{1}}-6 m_{\psi_{1}}^{2}+y^{2} v^{2} \cos ^{2} \beta}$
$g_{H \psi_{1} \psi_{1}}=\frac{1}{2} y^{2} v \sin 2 \beta \frac{m_{\psi_{1}}+M_{L} \sin 2 \theta}{2 M_{L}^{2}+4 M_{N} m_{\psi_{1}}-6 m_{\psi_{1}}^{2}+y^{2} v^{2} \cos ^{2} \beta}$

while for the Type-II model:

$$
\begin{aligned}
& g_{h \psi_{1} \psi_{1}}=\frac{\frac{1}{2} y^{2} v}{2 M_{L}^{2}+4 M_{N} m_{\psi_{1}}-6 m_{\psi_{1}}^{2}+\frac{1}{2} y^{2} v^{2}(1-\cos 2 \beta \cos 2 \theta)} \\
& \times\left(m_{\psi_{1}}(1+\cos 2 \beta \cos 2 \theta)+M_{L} \sin 2 \beta \sin 2 \theta\right) \\
& g_{H \psi_{1} \psi_{1}}=\frac{-\frac{1}{2} y^{2} v}{2 M_{L}^{2}+4 M_{N} m_{\psi_{1}}-6 m_{\psi_{1}}^{2}+\frac{1}{2} y^{2} v^{2}(1-\cos 2 \beta \cos 2 \theta)} \\
& \times m_{\psi_{1}} \sin 2 \beta \cos 2 \theta+M_{L} \cos 2 \beta \sin 2 \theta
\end{aligned}
$$

As evident, in the Type-I model, both the DM couplings with the CP-even bosons become zero in case that $m_{\psi_{1}}+$ $M_{L} \sin 2 \theta=0[18,20]$. This is actually a generic blind spot occurring in the case that all the new fermions couple to a same Higgs doublet. The case of the Type-II model is less trivial and the blind spot is obtained either by the condition for destructive interference:

$$
\begin{gathered}
\frac{\xi_{h}^{q}}{m_{h}^{2}}\left(m_{\psi_{1}}(1+\cos 2 \beta \cos 2 \theta)+M_{L} \sin 2 \beta \sin 2 \theta\right) \\
\quad=\frac{\xi_{H}^{q}}{m_{H}^{2}}\left(m_{\psi_{1}} \sin 2 \beta \cos 2 \theta+M_{L} \cos 2 \beta \sin 2 \theta\right)
\end{gathered}
$$

or in the case that the coupling of the DM with the SMlike Higgs is null, so $\left(m_{\psi_{1}}(1+\cos 2 \beta \cos 2 \theta)+M_{L} \sin 2 \beta\right.$ $\sin 2 \theta)=0$, while the other CP-even Higgs is heavy enough so that the corresponding contribution to the DD crosssection is not in tension with experimental limits.

An illustration of the occurrence of the blind spots, as function of $\tan \theta$ and some example assignations of the parameters, is shown in Fig. 3 for the Type-I (upper panel) and Type-II (lower panel) model. The plot represents indeed the ratio between the computed scattering cross-section and the limit on the cross-section, for the value of the DM mass corresponding to the considered parameters, as provided by XENON1T. The blind spot corresponds to a sharp decrease, from values eventually greater than 1 , implying an experimentally excluded configuration, of this ratio to values much below 1 , corresponding to an experimentally viable configuration. In the case of the Type-I model, in agreement with the previous discussion, the position of the blind spot is poorly sensitive to the assignation of $m_{H}$ and $y$. Since we have considered a value of $M_{N}$ sensitively lower than $M_{L}$, 

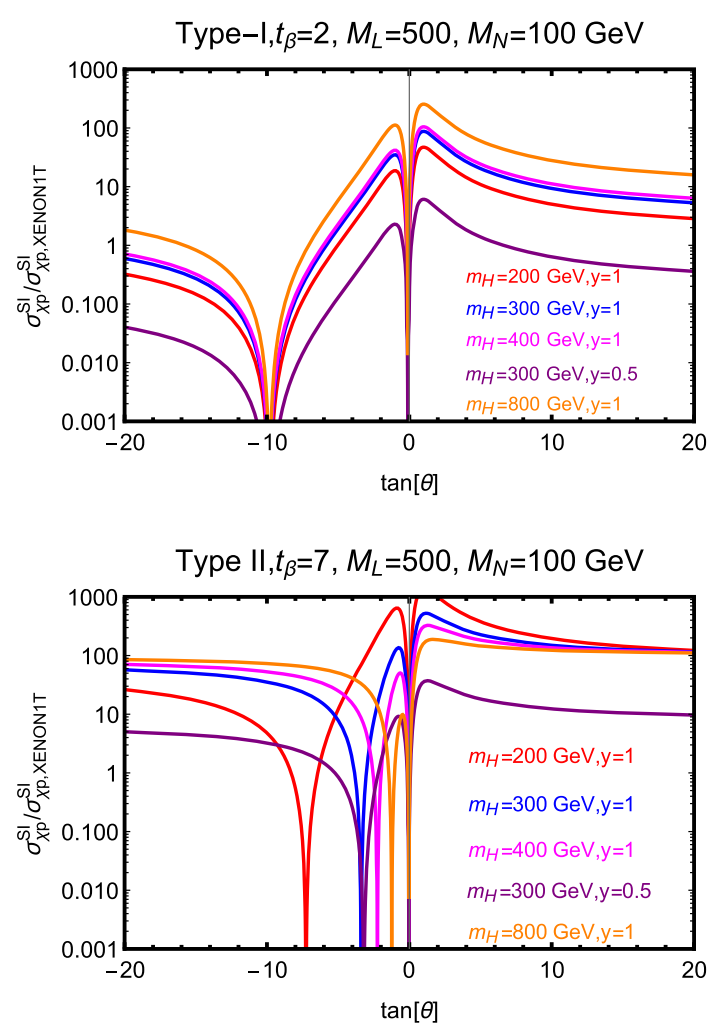

Fig. 3 Ratio of SI Direct Detection cross-section over the current limit value, as function of $\tan \theta$ for Type-I (upper), Type-II (bottom panel) models and for some assignations of $m_{H}$ and $y$, reported in the plots. In all cases we have fixed $M_{L}=500 \mathrm{GeV}$ and $M_{N}=100 \mathrm{GeV}$

we can safely take $m_{\psi_{1}} \simeq M_{N}$ and hence determine the blind spot through $M_{N}+M_{L} \sin 2 \theta=0$ which is solved for $\tan \theta=-0.1,-9.9$. On the contrary, in the case of the TypeII model, the position of the blind spot depends also on $m_{H}$ and there is no simple analytical expression for the value of $\tan \theta$ corresponding to it. In case of cancellation (or destructive interference) of the coupling of the DM with the CP-even Higgs bosons it might be necessary to take into account generally subdominant interactions like 29 . For the same reason, in the determination of the cross-section reported in Fig. 3, as well as throughout the rest of the paper, we have also included one-loop corrections to SI cross-section arising from interaction of the DM with the $Z$ and $W$ bosons [120-122] and, eventually, with the pseudoscalar boson $A$ [123-125] if this is light enough. As evidenced by the figure, even in presence of these corrections, it is possible to achieve a cross-section well below experimental limits for specific values of the angle $\theta$.

\subsection{Indirect detection}

As evidenced by the expressions provided in Appendix A, the DM annihilation channels into SM fermions and gauge bosons pairs feature $s$-wave, i.e. velocity independent, annihilation cross-sections. Thermal DM production can be thus tested, in our framework, also through Indirect Detection (ID). The most prominent signals come from $\gamma$-rays originating mainly from annihilations into $W W, Z Z, \bar{t} t, \bar{b} b$ and $\tau^{+} \tau^{-}$. Particularly interesting would be, in this context, the scenario of a light pseudoscalar since it would allow for a fit of the $\gamma$-ray Galactic Center (GC) excess [28,126,127]. DM interpretations of $\gamma$-ray signals are, nevertheless, challenged by the exclusion limits from absence of evidences in Dwarf Spheroidal Galaxies (DSph) [128] as well as, since recently, searches in the Milky-Way Halo away from the GC [129] (these exclude, in particular, the $\bar{b} b$ interpretation of the GC excess). In this work we will not attempt to provide a DM interpretation of the GC and focus, more conservatively, on the constraints on the DM annihilation cross-section.

At the moment Indirect Detection constraints can probe thermal DM production up to DM masses of around $100 \mathrm{GeV}$ and are rarely competitive with respect to Direct Detection constraints. In order to simplify the presentation of our results we will report Indirect Detection limits only when they are effectively complementary to other experimental searches while and omit them in the other cases.

\subsection{Invisible decays of the Higgs and of the Z}

In the setup under consideration the DM is coupled, in pairs, both to the Higgs and to the $\mathrm{Z}$ boson. In the case it is lighter then $m_{h} / 2, m_{Z} / 2$, an invisible decay channel for the latter becomes accessible. This possibility is however experimentaly disfavored [130]. We have hence imposed in our analysis that, when the processes are kinematically allowed, the invisible branching ratio of the Higgs fullfills the upper bound $B r(h \rightarrow$ inv $)<0.2$ while, for what concerns the invisible width of the $Z, \Gamma(Z \rightarrow$ inv $)<2.3 \mathrm{MeV}$ [26].

\subsection{LHC searches of the new fermions}

The new fermionic sector featured by our model might be probed also by collider searches. A detailed study goes, however, beyond the scopes of this paper. We will then illustrate qualitatively the most relevant searches and their potential impact on the parameter space of the model.

In the scenario under consideration at least some of new fermions feature sizeable couplings with the SM gauge bosons. They can be then produced, in proton collisions at LHC, through Drell-Yann processes of the type:

$$
p p \rightarrow \psi^{+} \psi^{-}, \quad p p \rightarrow \psi^{ \pm} \psi_{i}, \quad p p \rightarrow \psi_{i} \psi_{j}
$$

with the charged fermions subsequently decaying into a $W$ (either on or off shell) and a neutral fermion (typically the lightest one, i.e. the DM candidate) while the heavy neutral fermions features different possible decay channels, i.e. into a lighter one and a Z, or possibly, Higgs boson (in both cases 
the particles can be off-shell), or the charged fermion $\psi^{ \pm}$ and $\mathrm{a} \mathrm{W}$ boson. In the case of production processes involving neutral fermions, the different possible signals depend on the size of the elements of the mixing matrix $U$, determining the size of the hypercharge and doublet components of the $\psi_{i}$ states.

For what concerns detection prospects, the cleanest signatures are provided by production processes of the heavy fermions, other than the DM $\psi_{1}$, with subsequent decays involving $W / Z$ bosons, leading to events with missing energy accompagned by 2-4 charged leptons (see e.g. [20] for a classification of the possible processes). Among these processes the strongest constraints apply to $p p \rightarrow \psi_{i=2,3} \psi^{ \pm} \rightarrow$ $W^{ \pm} Z \psi_{1} \psi_{1}$, which leads to a 3 lepton plus missing energy signature. The corresponding limits from CMS and ATLAS searches (see [131,132] for the most recent) should be appropriately recasted in order to be applied for the scenario under consideration. Such recasting has been performed, for example, in [133] for the $\sqrt{s}=8 \mathrm{TeV}$ data sets $[134,135]$. It has been found there that these searches constrain the regions $m_{\psi_{2}}, m_{\psi_{3}}, m_{\psi^{ \pm}} \lesssim 270 \mathrm{GeV}$ and $m_{\psi_{1}} \lesssim 75 \mathrm{GeV}$. Notice that these constraints mostly apply to the case $M_{N}<M_{L}$. In the opposite case the lightest neutral fermions are mostly doublet like and are very close in mass with the charged state $\psi^{ \pm}$. This kind of configuration is more complicated to probe at the collider since it would correspond to the production of long-lived particles, leading to displaced vertices or being eventually detector stable.

The new fermions might also be produced in the decays of resonantly produced new Higgs bosons. In such a case it would be possible to the test directly the production of DM pairs by looking for events with missing energy accompagned by initial state radiation, i.e. monojet. Recasting the corresponding limits $[136,137]$, goes beyond the scopes of this work and will be left to future study (see e.g. [138] for an example of this recasting on a simplified model).

We finally remind that the mass of the charged new fermions $m_{\psi^{ \pm}} \simeq M_{L}$ is also constrained by LEP [139]. For this reason in all the plots shown below, only regions with $M_{L} \gtrsim 100 \mathrm{GeV}$ are visualized.

\subsection{Sommerfeld enhancement for relic density and Indirect Detection}

A complete treatment of the DM phenomenology would require the inclusion of the Sommerfeld enhancement for the DM annihilation cross-section. The Sommerfeld enhancement consists into an enhancement of the DM annihilation cross-section at low relative velocity, due to the presence of an attractive long-range potential, typically induced by the multiple exchange of light (with respect to the DM) mediators. In the case in which the DM is, at least partially, charged under the EW interactions, a sizeable Sommerfeld enhancement might be produced by loop effects from exchange of electroweak bosons, when the DM mass is of the order or above the $\mathrm{TeV}$ scale. The associated corrections to the DM relic density have been determined in the limit of a pure $S U(2)$ nplet DM in [140-142] and subsequently revised and corrected in $[143,144]$. In case of doublet like DM these corrections have been found to be rather small, hence corresponding to a slight (non-neglible though) increase of the value of the mass (in case determined mostly by $M_{L}$ ), with respect to the one quoted in Sect. 4.1, corresponding to the correct relic density. The computation of the Sommerfeld enhancement in the case of mixed (singled-doublet in our scenario) DM is typically extremely complicated as consequence of its strong dependence on the mass splitting between the DM and the lightest charged and neutral states of the new fermionic spectrum. This kind of computation has been tackled mostly in SUSY inspired scenarios, see e.g. [145-147]. More recently more general scenarios have been considered though [144]. The detailed determination of the Sommerfeld correction in the model under study is not in the scopes of this paper. Given the already limited impact on the relic density in the pure $S U$ (2) limit we expect anyway a marginal variation to our results from the inclusion of Sommerfeld corrections.

Besides the gauge bosons, the scenario under study features two potential light force carriers, being the SM-like Higgs boson $h$ and the light pseudoscalar $A$. The Sommerfeld enhanchement associated to $h$ has been considered in the case of the Higgs portal model [148] while studies for a generic pseudoscalar mediator can be found e.g. in $[149,150]$. Similarly to the case of the gauge bosons, Sommerfeld enhancement induced by exchange of $h$ is expected to be relevant for DM masses well above the $\mathrm{TeV}$ scale, marginally considered in our study. Less trivial would be instead the case of the pseudoscalar. As evidenced by Fig. 6, nevertheless, we have considered the case of a light pseudoscalar (we remind that in our setup it cannot be lighter than around $60 \mathrm{GeV}$ in order to comply with collider bounds) in relation to comparatively light DM masses, not above $300 \mathrm{GeV}$; consequently, Sommerfeld enhancement can be safely neglected.

Being a low velocity effect, Sommerfeld enhancement can impact Indirect Detection to a sensitively larger extent with respect to relic density. Weak constrains are, however, expected in the case of annihilation into scalar and pseudoscalar final states, being them p-wave processes, and, hence, they have been neglected. This might not the case, instead, for annihilations into gauge boson final states. Sommerfeld enhancement can give rise, in particular, to strong $\gamma$-ray signals, see e.g [142,151-154]. While this effect is particularly pronounced for DM belonging to high $S U$ (2) multiplets, namely $n \geq 3$, it is modest, instead, in the case of a $S U(2)$ doublet $[140,155,156]$ unless one considers DM masses of around $7 \mathrm{TeV}$, well outside the range adopted in 

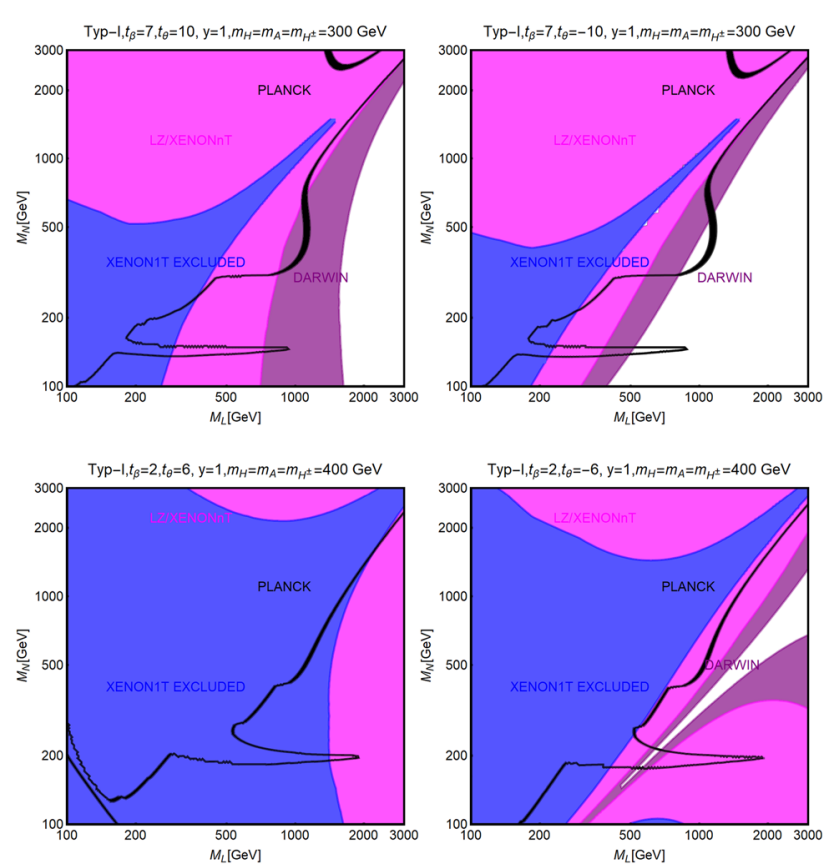

Fig. 4 Comparison between relic density constraints and limit/projected sensitivities, in the bidimensional plane $\left(M_{L}, M_{N}\right)$ in the Type-I model (abbreviated Typ-I) for some assignations of $\theta, \tan \beta$ and $m_{H, A, H^{ \pm}}$, reported on top of the panel. In each panel the black curve represents the isocontour of the correct relic density while the blue region is excluded by current constraints from XENON1T. The magenta and purple regions will be ruled out in case of null signals from, respectively, XENONnT/LZ and DARWIN

this study. ${ }^{10}$ We hence expect that Sommerfeld enhancement does not sensitively affect ID prospects of our scenario.

\section{Results and discussion}

We have now all the elements for examining in detail the constraints on the model under consideration. We show first of all in Figs. 4 and 5 the interplay between relic density and Direct Detection, in the bidimensional plane $\left(M_{L}, M_{N}\right)$, for, respectively, Type-I and Type-II scenarios. We have, again, focussed on some specific assignations of the other parameters of the theory and assumed, for simplicity, degenerate masses for the new bosons as well as the alignment limit. As we will see, constraints from Direct Detection are extremely strong, hence we focussed on $M_{N}, M_{L}>100 \mathrm{GeV}$ in order to avoid the regions of maximal sensitivity for these experiments $\left(M_{L}<100 \mathrm{GeV}\right.$ would be in any case forbidden by LEP limits on production of new charged particles). In each plot the parameter space corresponding to the correct relic density, represented by the black isocontours, is compared with the

\footnotetext{
10 Sizable Sommerfeld corrections for DM masses of the order of the $\mathrm{TeV}$ could be achieved for mass splittings well below the one typically expected from radiative corrections, as could occur in some specific SUSY realizations [156].
}
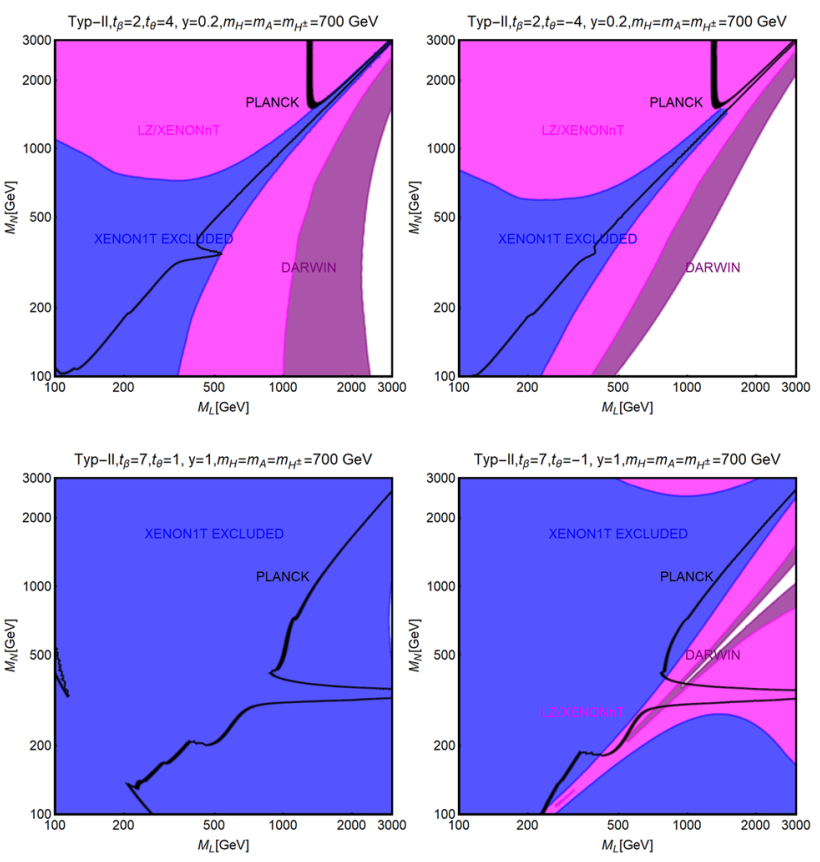

Fig. 5 The same as Fig. 4 but for Type-II model (abbreviated Typ-II) and different parameter assignations

excluded region (blue) by current limits from Direct Detection, essentially determined by XENON1T [110], as well as the projected sensitivities from XENONnT/LZ [113,114] (magenta, given the similar sensitivity we are assuming the same projected excluded region for both experiments) and DARWIN [115] (purple).

All the panels of Figs. 4 and 5 are grouped in pairs differing by the sign of $\tan \theta$, in order to highlight the effect of the blind spots described in Sect. 4.2, since they occur only for negative $\tan \theta$.

In the case of the Type-I model the two pairs of benchmarks are mostly differentiated by the assignment of $\tan \theta$ and $\tan \beta$. As evidenced by the upper panels of Fig. 4, the moderate value of $\tan \beta$ corresponds to a suppression of the interactions of the DM with the SM fermions, affecting mostly the DD bounds, which are substantially weaker with respect to the case $\tan \beta=2$. As already discussed in Sect. 4.2 the blind spots for the Type-I model can be described by the simple equation $M_{N}+M_{L} \sin 2 \theta=0$. For $\tan \theta=$ $-10(-6)$ a blind spot is achieved for $M_{L} / M_{N}=5(3)$. In the first case the blind spot affects projected excluded regions by XENONnT/LZ and DARWIN while it leaves the current excluded region mostly unchanged. Viable relic density is achieved, for $y=1$ and $m_{\psi_{1}} \gtrsim 300 \mathrm{GeV}$, without relying on blind spots, thanks to the annihilations into the Higgs states. For the pair of benchmarks reported on the lower panels of Fig. 4, on the contrary, the blind spot weakens also the current exclusion bounds and its presence is necessary to achieve the correct DM relic density over a sizeable portion of the parameter space. This viable parameter space would be nev- 
ertheless almost completely ruled out by a negative signal at XENONnT/LZ.

A similar analysis, for two pairs of Type-II benchmarks, is shown in Fig. 5. Contrary to the Type-I model we have chosen a heavier mass of the Higgs mediators, $700 \mathrm{GeV}$ in all panels, in order to comply with the observational constraints discussed in the previous sections. The two pairs of benchmarks are distinguished by the assignations of $y$, namely 0.2 and 1 for, respectively the upper and the lower panels as well as by the assignations of $\tan \beta$ and $\tan \theta$. For the chosen parameter assignations, the blind spot correspond, in good approximation, to the condition $g_{h \psi_{1} \psi_{1}}=0$, fulfilled for $M_{L} / M_{N} \approx 4.1,3.5$ for, respectively, the upper and lower panels. As evidenced by the plots, the Type-II model is more constrained. As shown by the upper panels of Fig. 5, viable relic density is achieved, without relying on blind spots, by lowering the value of $y$ to 0.2 . On the contrary, by taking $y=1$ and a moderate value of $\tan \beta$, for positive $\tan \theta$ (implying absence of any blind spot) the whole range of considered values of $M_{N}, M_{L}$ is already excluded by present bounds from DD. By considering negative $\tan \theta$ a narrow viable region around the s-channel resonance opens. It will be anyway ruled out in absence of signals at next future Direct Detection experiments.

The Type-I model offers another attractive possibility to evade Direct Detection constraints consisting into a light CPodd boson $A$. In such a case, indeed, it is possible to achieve a sizable s-wave dominated annihilation cross-section of the DM into SM fermions, without strong additional contribution to the scattering cross-section since interactions mediated by a pseudoscalar are momentum suppressed (at least at the tree level). The DM annihilation cross-section can be also enhanced by the presence of $h A, A A$ and $Z A$ final states. Moreover the presence of a velocity independent cross-section would allow ID as complementary probe and possibly fit the GC excess $[28,127]$. Sizeable constraints from absence $\gamma$-ray signals from DSph would be present though.

We have then shown in Fig. 6, the combination of the DM constraints, for some parameter assignations, in the case of a light pseudoscalar Higgs $A$. Differently to Figs. 4, 5 we have focussed on the case of a light mostly singlet like DM by considering for $M_{N}, M_{L}$ the ranges $10 \leq M_{N} \leq 300 \mathrm{GeV}$ and $100 \leq M_{L} \leq 1000 \mathrm{GeV}$. The isocontours of the correct relic density feature multiple "cusps" corresponding to the "poles", $m_{\psi_{1}} \simeq \frac{m_{h}}{2}, m_{\psi_{1}} \simeq \frac{m_{Z}}{2}, m_{\psi_{1}} \simeq \frac{m_{h}}{2}, m_{\psi_{1}} \simeq \frac{m_{A}}{2}$ and $m_{\psi_{1}} \simeq \frac{m_{H}}{2}$. On the contrary, the isocontours describing the limit from DSph feature only the cusp associated to the $m_{A}$ resonance, since only the s-channel exchange of the pseudoscalar Higgs leads to a s-wave dominated cross-section (notice that the s-channel exchange of the $Z$-boson gives a s-wave but helicity suppressed contribution, see Appendix A for details), hence sensitive to ID.
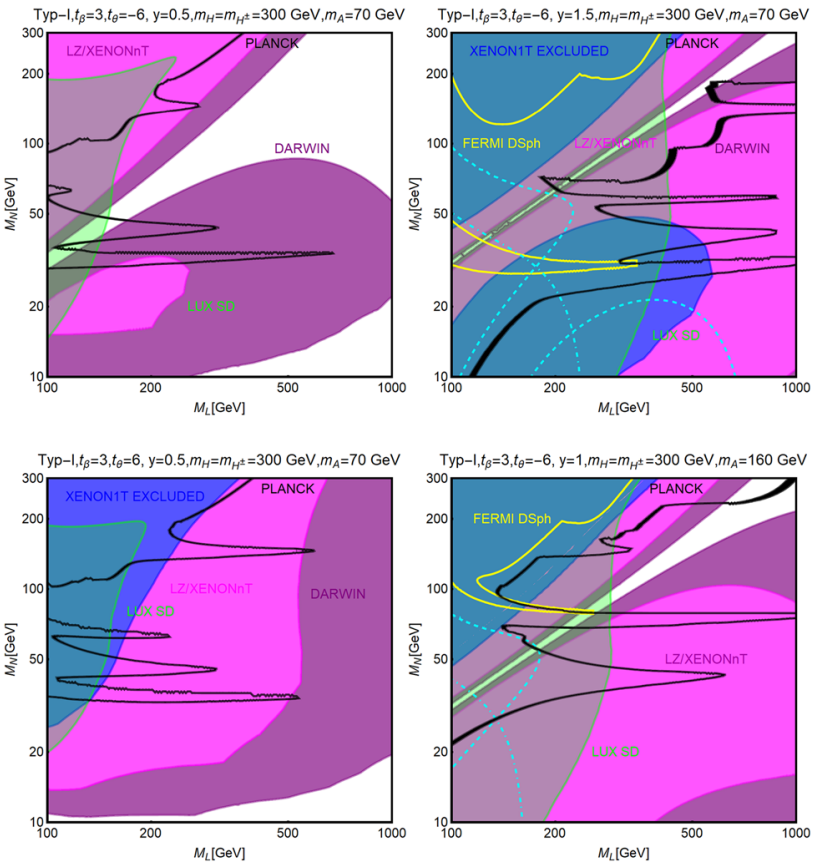

Fig. 6 Summary of DM constraints for some realizations of the Type-I model (Typ-I in the label of the panels) with low mass pseudoscalar Higgs. As usual, the black contours correspond to the correct relic density while the blue/magenta/purple regions correspond to the present/future exclusions by XENON1T/XENONnT/DARWIN. In addition we have reported in green the excluded regions from SD limits, as provided by LUX. In the right panels the regions within the dashed (dot-dashed) cyan lines are excluded by the constraints on the invisible width of the Higgs $(Z)$ boson while the regions inside the yellow contours are excluded by constraints from searches of $\gamma$-rays in DSph by FERMI

As evident, the low mass mediator allows to achieve the correct relic density for DM masses below $100 \mathrm{GeV}$ (we notice anyway that the regions $M_{N}<M_{L}, M_{L} \lesssim 300 \mathrm{GeV}$ are sensitive to LHC searches of the new fermions). At these low values, constraints from SI interactions are complemented by one from SD interactions (green regions in the plot) as well as from the invisible decay width of the $h$ and $Z$ bosons. Given the $1 / \tan \beta$ suppression of the coupling of the extra Higgs bosons with SM fermions, Indirect Detection cannot efficiently probe the scenario under consideration unless values of $y$ above 1 (see second panel of Fig. 6 are considered).

Regions of parameters space complying with all observational constraints are nevertheless present. These regions will be, however, fully probed and possibly ruled out by forthcoming Direct Detection experiments.

\section{Conclusions}

We have performed an extensive analysis of the DM phenomenology of a model with singlet-doublet Dark Matter coupled with a two doublet Higgs sector. We have considered 
two scenarios, globally dubbed as Type-I and Type-II model, for the couplings of SM and new fermions with the Higgs doublets. In all cases the most competitive constraints come from limits from Direct Detection. In the case of the TypeII model these can be evaded only by invoking parameter assignations inducing blind-spots in the couplings responsible for Direct Detection. In the case of the Type-I model it is instead possible to evade Direct Detection constraints even without relying on blind spots. The Type-I model presents the additional interesting possibility of a light pseudoscalar Higgs boson.

For all the considered scenarios, next future Direct Detection facilities will fully probe the viable region for thermal DM relic density.

Acknowledgements We thank Federico Mescia and Olcyr Sumensari for the fruitful discussions. We are also indebted with Luca di Luzio for the valuable comments on the draft.

Open Access This article is distributed under the terms of the Creative Commons Attribution 4.0 International License (http://creativecomm ons.org/licenses/by/4.0/), which permits unrestricted use, distribution, and reproduction in any medium, provided you give appropriate credit to the original author(s) and the source, provide a link to the Creative Commons license, and indicate if changes were made. Funded by SCOAP ${ }^{3}$.

\section{A Approximate analytical expressions for the DM annihilation cross-section}

We summarize, in this appendix, analytical approximations for the cross-sections of the most relevant, for our study, annihilation channels of the DM. These have been obtained by considering the so-called velocity expansion $\langle\sigma v\rangle \simeq a+$ $b v_{\psi}$ and by taking, for each annihilation channel, only the leading contribution.

We start with the $\bar{f} f$ channel:

$$
\begin{aligned}
\langle\sigma v\rangle_{f f}= & \frac{1}{2 \pi} \sum_{f} n_{c}^{f} \sqrt{1-\frac{m_{f}^{2}}{m_{\psi_{1}}^{2}}}\left[\frac{\left|\xi_{A}^{f}\right|^{2}\left|y_{A \psi_{1} \psi_{1}}\right|^{2} m_{f}^{2} m_{\psi_{1}}^{2}}{v^{2}\left(4 m_{\psi_{1}}^{2}-m_{A}^{2}\right)^{2}}\right. \\
& +\frac{m_{f}^{2}}{m_{Z}^{4}}\left|g_{Z \psi_{1} \psi_{1}}^{A}\right|^{2}\left|g_{Z f f}^{A}\right|^{2}-2 \frac{m_{f}^{2} m_{\psi_{1}}}{v m_{Z}^{2}\left(4 m_{\psi_{1}}^{2}-m_{A}^{2}\right)} \\
& \left.\times \operatorname{Re}\left[\left(\xi_{A}^{f}\right)^{*}\left(y_{A \psi_{1} \psi_{1}}\right)^{*} g_{Z \psi_{1} \psi_{1}}^{A} g_{Z f f}^{A}\right]\right]
\end{aligned}
$$

As evident, the $s$-wave term receives contributions only from s-channel exchange of the pseudoscalar Higgs $A$ and of the $Z$ boson with the latter being, however, helicity suppressed and, hence, relevant only for DM masses close to the mass of the top-quark.
In the regime $m_{\psi_{1}}<m_{H, A, H^{ \pm}}$the other relevant annihilation channels are in the $W W, Z Z$ and $Z h$ final states. Their cross-sections are given by:

$$
\begin{aligned}
& \langle\sigma v\rangle_{W W}=\frac{1}{4 \pi} \sqrt{1-\frac{m_{W}^{2}}{m_{\psi_{1}}^{2}}} \frac{1}{m_{W}^{4}\left(m_{W}^{2}-m_{\psi_{1}}^{2}-m_{\psi^{ \pm}}^{2}\right)^{2}} \\
& \times\left[\left(\left|g_{W \psi_{1}}^{V}\right|^{2}+\left|g_{W \psi_{1}}^{A}\right|^{2}\right)^{2}\left(2 m_{W}^{4}\left(m_{\psi_{1}}^{2}-m_{W}^{2}\right)\right)\right. \\
& \left.\left.+2\left|g_{W \psi_{1}}^{V}\right|^{2}\left|g_{W \psi_{1}}^{A}\right|^{2} m_{\psi^{ \pm}}^{2}\left(4 m_{\psi_{1}}^{4}+3 m_{W}^{2}-4 m_{\psi_{1}}^{2} m_{W}^{2}\right)\right)\right] \\
& \langle\sigma v\rangle_{Z Z}=\frac{1}{4 \pi} \sqrt{1-\frac{m_{Z}^{2}}{m_{\psi_{1}}^{2}}} \sum_{i=1,3} \frac{1}{\left(m_{Z}^{2}-m_{\psi_{1}}^{2}-m_{\psi_{i}}^{2}\right)^{2}} \\
& \times\left(\left|g_{Z \psi_{1} \psi_{i}}^{V}\right|^{2}+\left|g_{Z \psi_{1} \psi_{i}}^{A}\right|^{2}\right)\left(\left|g_{Z \psi_{1} \psi_{j}}^{V}\right|^{2}+\left|g_{Z \psi_{1} \psi_{j}}^{A}\right|^{2}\right)\left(m_{\psi_{1}}^{2}-m_{Z}^{2}\right) \\
& \langle\sigma v\rangle_{Z h}=\frac{1}{\pi} \sqrt{1-\frac{\left(m_{h}+m_{Z}\right)^{2}}{4 m_{\psi_{1}}^{2}}} \sqrt{1-\frac{\left(m_{h}-m_{Z}\right)^{2}}{4 m_{\psi_{1}}^{2}}} \\
& \times\left[\frac { | \lambda _ { h A Z } | ^ { 2 } | y _ { A \psi _ { 1 } \psi _ { 1 } } | ^ { 2 } } { m _ { Z } ^ { 2 } ( 4 m _ { \psi _ { 1 } } ^ { 2 } - m _ { A } ^ { 2 } ) ^ { 2 } } \left(m_{\psi_{1}}^{4}-\frac{1}{2} m_{\psi_{1}}^{2}\left(m_{h}^{2}+m_{Z}^{2}\right)\right.\right. \\
& \left.+\frac{1}{16}\left(m_{h}^{2}-m_{Z}^{2}\right)^{2}\right) \\
& \times \frac{1}{256 m_{\psi_{1}}^{2} m_{Z}^{6}} \lambda_{h Z Z}^{2}\left|g_{Z \psi_{1} \psi_{1}}^{A}\right|^{2} \\
& \times\left(m_{h}^{4}+\left(m_{Z}^{2}-4 m_{\psi_{1}}^{2}\right)^{2}-2 m_{h}^{2}\left(m_{Z}^{2}-4 m_{\psi_{1}}^{2}\right)\right) \\
& -\frac{1}{2} \operatorname{Re}\left[\lambda_{h A Z}^{*} y_{A \psi_{1} \psi_{1}}^{*} \lambda_{h Z Z} g_{Z \psi_{1} \psi_{1}}^{A}\right] \frac{1}{m_{\psi_{1}} m_{Z}^{4}\left(4 m_{\chi}^{2}-m_{A}^{2}\right)} \\
& \left.\times\left(m_{\psi_{1}}^{4}-\frac{1}{2} m_{\psi_{1}}^{2}\left(m_{h}+m_{Z}\right)^{2}+\left(m_{h}^{2}-m_{Z}^{2}\right)^{2}\right)\right]
\end{aligned}
$$

where the trilinear couplings used above are given by [33]:

$$
\begin{aligned}
& \lambda_{h Z Z}=\frac{2 m_{Z}^{2}}{v} \sin (\alpha-\beta) \\
& \lambda_{h A Z}=\frac{m_{Z}}{v} \cos (\alpha-\beta) \\
& \lambda_{H A Z}=\frac{m_{Z}}{v} \sin (\alpha-\beta)
\end{aligned}
$$

The s-wave contributions to the $W W$ and $Z Z$ cross-sections are mostly determined by $\mathrm{t}$-channel exchange of the new neutral and charged fermions, s-channel exchange of the Higgs states is present only in the velocity dependent term. The annihilation cross-section into $Z h$ receives an additional contribution, with respect to the "minimal" singlet-doublet model, from s-channel exchange of the pseudoscalar Higgs A.

In the case that the pseudoscalar Higgs $A$ is lighter than the DM the $Z A, h A$ and $A A$ final states can contribute in a relevant way to the DM relic density. The corresponding cross-sections are given by: 


$$
\begin{aligned}
& \langle\sigma v\rangle_{Z A}=\frac{v_{\psi}^{2}}{16 \pi m_{Z}^{2}} \sqrt{1-\frac{\left(m_{A}-m_{Z}\right)^{2}}{4 m_{\psi_{1}}^{2}}} \sqrt{1-\frac{\left(m_{A}+m_{Z}\right)^{2}}{4 m_{\psi_{1}}^{2}}} \\
& \times\left(16 m_{\psi_{1}}^{4}-8 m_{\psi_{1}}^{2}\left(m_{Z}^{2}+m_{A}^{2}\right)+\left(m_{Z}^{2}-m_{A}^{2}\right)^{2}\right) \\
& \times\left[\frac{\lambda_{h A Z} y_{h \psi_{1} \psi_{1}}}{\left(4 m_{\psi_{1}}^{2}-m_{h}^{2}\right)}+\frac{\lambda_{H A Z} y_{H \psi_{1} \psi_{1}}}{\left(4 m_{\psi_{1}}^{2}-m_{H}^{2}\right)}\right]^{2}, \\
& \langle\sigma v\rangle_{h A}=\frac{1}{16 \pi} \sqrt{1-\frac{\left(m_{h}+m_{A}\right)^{2}}{4 m_{\psi_{1}}^{2}}} \sqrt{1-\frac{\left(m_{h}-m_{A}\right)^{2}}{4 m_{\psi_{1}}^{2}}} \\
& \times\left[\frac{\lambda_{h A A}^{2} y_{A \psi_{1} \psi_{1}}^{2}}{\left(4 m_{\psi_{1}}^{2}-m_{A}^{2}\right)^{2}}+\frac{1}{4} \frac{\left.\lambda_{h A Z}^{2} g_{Z \psi_{1} \psi_{1}}^{2}\left(m_{A}^{2}-m_{h}^{2}\right)^{2}\right)}{\left(4 m_{\psi_{1}}^{2}-m_{Z}^{2}\right)^{2}}\right. \\
& \times \sum_{i, j=1,3} \frac{y_{A \psi_{1} \psi_{i}} y_{A \psi_{1} \psi_{j}}^{*} y_{h \psi_{1} \psi_{i}} y_{h \psi_{1} \psi_{j}}^{*}}{m_{\psi_{1}}^{2}\left(m_{A}^{2}+m_{h}^{2}-2 m_{\psi_{1}}^{2}-m_{\psi_{i}}^{2}\right)^{2}\left(m_{A}^{2}+m_{h}^{2}-2 m_{\psi_{1}}^{2}-m_{\psi_{j}}^{2}\right)^{2}} \\
& \times\left(m_{A}^{4}+m_{h}^{4}-8 m_{\psi_{1}} m_{\psi_{j}} m_{h}^{2}+16 m_{\psi_{i}} m_{\psi_{j}} m_{\psi_{1}}^{2}\right. \\
& \left.-2 m_{A}^{2}\left(m_{h}^{2}-4 m_{\psi_{1}} m_{\psi_{j}}\right)\right) \\
& \operatorname{Re}\left[\lambda_{h A A}^{*} y_{A \psi_{1} \psi_{1}}^{*} y_{h \psi_{1} \psi_{1}}^{*} \lambda_{h A Z} g_{Z \psi_{1} \psi_{1}}^{A}\right] \frac{\left(m_{A}^{2}-m_{h}^{2}\right)}{m_{Z}^{2} m_{\psi_{1}}} \\
& +\frac{2}{m_{\psi_{1}}^{2}} \operatorname{Re}\left[\lambda_{h A A}^{*} y_{A \psi_{1} \psi_{1}}^{*} y_{h \psi_{1} \psi_{1}}^{*} y_{h \psi_{1} \psi_{i}} y_{A \psi_{1} \psi_{i}}\right] \\
& \times \frac{\left(m_{A}^{2} m_{\psi_{1}}-m_{h}^{2} m_{\psi_{1}}+4 m_{\psi_{i}} m_{\psi_{1}}^{2}\right)}{\left(m_{A}^{2}+m_{h}^{2}-2 m_{\psi_{1}}^{2}-2 m_{\psi_{i}}^{2}\right)\left(4 m_{\psi_{i}}^{2}-m_{A}^{2}\right)} \\
& +\frac{1}{2} \sum_{i=1,3} \operatorname{Re}\left[\lambda_{h A Z}^{*} g_{Z \psi_{1} \psi_{1}}^{*} y_{h \psi_{1} \psi_{i}} y_{A \psi_{1} \psi_{i}}\right] \\
& \left.\times \frac{\left(m_{A}^{2}-m_{h}^{2}\right)^{2}+4 m_{\psi_{1}} m_{\psi_{i}}\left(m_{A}^{2}-m_{h}^{2}\right)}{m_{\psi_{1}}^{2} m_{Z}^{2}\left(m_{A}^{2}+m_{h}^{2}-2 m_{\psi_{1}}^{2}-2 m_{\psi_{i}}^{2}\right)}\right]
\end{aligned}
$$

and:

$$
\begin{aligned}
\langle\sigma v\rangle_{A A}= & \frac{v_{\psi}^{2}}{128 \pi} \sqrt{1-\frac{m_{A}^{2}}{m_{\psi}^{2}}} \\
& \times\left[\left[\frac{\lambda_{A A h} y_{h \psi_{1} \psi_{1}}}{\left(4 m_{\psi_{1}}^{2}-m_{h}^{2}\right)}+\frac{\lambda_{A A H} y_{H \psi_{1} \psi_{1}}}{\left(m_{\psi_{1}}^{2}-m_{H}^{2}\right)}\right]^{2}\right. \\
& +\frac{16}{3}\left|y_{A \psi_{1} \psi_{1}}\right|^{2} \frac{m_{\psi_{1}}^{2}\left(m_{\psi_{1}}^{2}-m_{A}^{2}\right)^{2}}{\left(2 m_{\psi_{1}}^{2}-m_{A}^{2}\right)^{4}} \\
& -\frac{8}{3}\left|y_{A \psi_{1} \psi_{1}}\right|^{2} \frac{m_{\psi_{1}}\left(m_{\psi_{1}}^{2}-m_{A}^{2}\right)}{\left(2 m_{\psi_{1}}^{2}-m_{A}^{2}\right)^{2}} \\
& \left.\times\left[\frac{y_{h \psi_{1} \psi_{1}} \lambda_{h A A}}{\left(4 m_{\psi_{1}}^{2}-m_{h}^{2}\right)}+\frac{y_{H \psi_{1} \psi_{1}} \lambda_{H A A}}{\left(4 m_{\psi_{1}}^{2}-m_{H}^{2}\right)}\right]\right]
\end{aligned}
$$

where:

$$
\begin{aligned}
\lambda_{h A A}= & -\frac{1}{4 v \sin 2 \beta}\left\{[\cos (\alpha-3 \beta)+3 \cos (\alpha+\beta)] m_{h}^{2}\right. \\
& \left.-4 \sin 2 \beta \sin (\alpha-\beta) m_{A}^{2}-4 \cos (\alpha+\beta) M^{2}\right\} \\
\lambda_{H A A}= & -\frac{1}{4 v \sin 2 \beta}\left\{[\sin (\alpha-3 \beta)+3 \sin (\alpha+\beta)] m_{H}^{2}\right.
\end{aligned}
$$

$$
\left.+4 \sin 2 \beta \cos (\alpha-\beta) m_{A}^{2}-4 \sin (\alpha+\beta) M^{2}\right\}
$$

Despite the velocity suppression $\left(v_{\psi}^{2}\right)$ the $A A$ and $Z A$ channels can provide not negligible contribution because of the sizeable trilinear scalar couplings.

\section{B Couplings of DM with the Higgs bosons}

As already shown in Sect. 3.2 the generic Lagrangian describing the interactions of the new fermions with the two Higgs doublets is (for simplicity we omit this time the mass terms):

$\mathcal{L}=-y_{i}^{L} L_{L} H_{i} N^{\prime}-y_{i}^{R} \bar{N}^{\prime} \tilde{H}_{i}^{\dagger} L_{R}+$ h.c.,

$i=1,2$

As already mentioned it is not appropriate to couple each new fermion arbitrarily with both Higgs doublets. This leaves four possible set of assignations of the couplings $y_{i}^{L, R}$. Throughout this work we have adopted two of these assignations which, combined with two specific assignations of the couplings of $H_{1,2}$ with the SM fermions, have been used to define the models dubbed as Type-I and Type-II. From a bottom-up perspective the choice of the coupling combinations for the new and the SM fermions is substantially free. Consequently the choice of focussing on just two combinations was mostly dictated by definiteness and simplicity.

In this appendix we will briefly discuss about potential changes induced by the choice of different combinations of the couplings as the ones considered in the main text. Leaving to future work the case of the couplings of the Higgs bosons with the SM fermions we will compare here the four possible assignations for the couplings of the SM singlet $N^{\prime}$ and the $\mathrm{SU}(2)$ doublets $L_{L, R}$ with the two Higgs doublets $H_{1}, H_{2}$ while considering, for all four cases, the same configuration, the Type-II, for the couplings of the Higgs doublets with the SM fermions.

As evidenced in the main text the viable regions for DM phenomenology are mostly determined by the interplay between relic density and (SI) Direct Detection. Concerning the latter, limits are in general very strong, especially when the coupling $y$ is of order 1 . It is hence important to identify for which assignations of the model parameters the blind spots occur. As already pointed in the text, and firstly shown in [28], in the case in which the new fermions are coupled only to one Higgs doublet, irrespective whether this is $H_{1}$ or $H_{2}$, a blind spot occurs when both couplings $g_{h \psi_{1} \psi_{1}}$ and $g_{H \psi_{1} \psi_{1}}$ become zero. In all cases this occurs when the condition $M_{N}+M_{L} \sin 2 \theta=0$ is fulfilled. In this regard, one of the omitted configurations has been actually accounted in our study. In the case in which the new fermions are selectively 

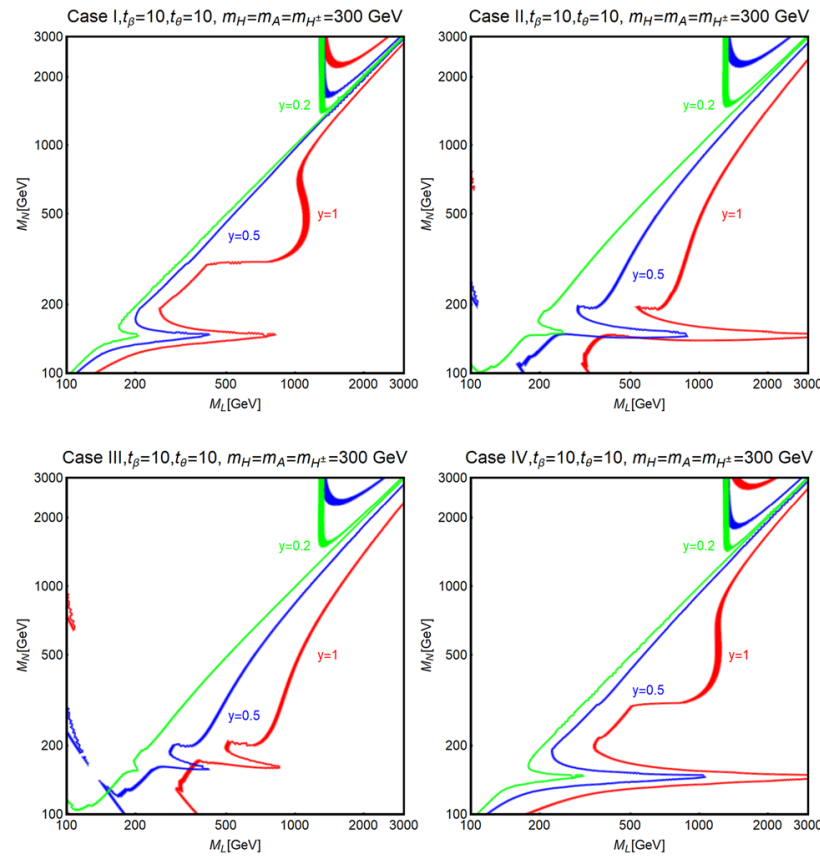

Fig. 7 Isocontours, in the bidimensional plane $\left(M_{L}, M_{N}\right)$ of the correct DM relic density for three assignations of $y$, namely $0.2,0.5,1$, and for fixed values of the model parameters, as reported on top of the panels. The four panels correspond to the four possible combinations, dubbed Case I-IV, of the couplings of the new fermions with the two Higgs doublets $H_{1,2}$ (see discussion in the text)

coupled with both Higgs doublets, instead, there is no simple analytical description of the blind spots. A comparison of the two possible cases would then require a dedicated study.

Concerning the relic density an analytical description would be similarly difficult because of the different annihilation channels for the DM.

A partial insight is provided by Fig. 7. Similarly to what done in Fig. 2, the isocontours of the correct relic density have been drawn for the three values $0.2,0.5$ and 1 of the coupling $y$ while all the other model parameters have been kept fixed. The four panels of the plot correspond to the four possible configurations for the couplings of the new fermionic sector. The assignations adopted for the Type-I and Type-II models studied in this work have been called Case I and Case II respectively (notice that this time the assignation for the coupling of the SM fermions with the Higgs doublets is the same though). The panel dubbed Case III describes the scenarios in which the new fermions couple only with the $\mathrm{H}_{2}$ doublet; Case IV represent finally the "opposite" coupling configurations as Case II. As evident, while the four panels are qualitatively different, by comparing the upper panels (analogous to the case of study of this work) and the lower panels of Fig. 7, no new feature seems to emerge in the latter. Despite a definitive assessment would require a more systematic study, there is no evidence that potentially relevant cases have been missed.

\section{References}

1. Planck, P. A. R. Ade et al., Planck 2015 results. XIII. Cosmological parameters. arXiv:1502.01589 [astro-ph.CO]

2. V. Silveira, A. Zee, SCALAR PHANTOMS. Phys. Lett. 161B, 136-140 (1985)

3. J. McDonald, Gauge singlet scalars as cold dark matter. Phys. Rev. D 50, 3637-3649 (1994). arXiv:hep-ph/0702143 [HEP-PH]

4. C.P. Burgess, M. Pospelov, T. ter Veldhuis, The Minimal model of nonbaryonic dark matter: A Singlet scalar. Nucl. Phys. B 619, 709-728 (2001). arXiv:hep-ph/0011335 [hep-ph]

5. Y.G. Kim, K.Y. Lee, The Minimal model of fermionic dark matter. Phys. Rev. D 75, 115012 (2007). arXiv:hep-ph/0611069 [hep-ph]

6. S. Andreas, C. Arina, T. Hambye, F.-S. Ling, M.H.G. Tytgat, A light scalar WIMP through the Higgs portal and CoGeNT. Phys. Rev. D 82, 043522 (2010). arXiv:1003.2595 [hep-ph]

7. S. Kanemura, S. Matsumoto, T. Nabeshima, N. Okada, Can WIMP Dark Matter overcome the Nightmare Scenario? Phys. Rev. D 82, 055026 (2010). arXiv:1005.5651 [hep-ph]

8. O. Lebedev, H.M. Lee, Y. Mambrini, Vector Higgs-portal dark matter and the invisible Higgs. Phys. Lett. B 707, 570-576 (2012). arXiv:1111.4482 [hep-ph]

9. Y. Mambrini, Higgs searches and singlet scalar dark matter: Combined constraints from XENON 100 and the LHC. Phys. Rev. D 84, 115017 (2011). arXiv:1108.0671 [hep-ph]

10. A. Djouadi, O. Lebedev, Y. Mambrini, J. Quevillon, Implications of LHC searches for Higgs-portal dark matter. Phys. Lett. B 709, 65-69 (2012). arXiv:1112.3299 [hep-ph]

11. L. Lopez-Honorez, T. Schwetz, J. Zupan, Higgs portal, fermionic dark matter, and a Standard Model like Higgs at $125 \mathrm{GeV}$. Phys. Lett. B 716, 179-185 (2012). arXiv:1203.2064 [hep-ph]

12. A. Djouadi, A. Falkowski, Y. Mambrini, J. Quevillon, Direct Detection of Higgs-Portal Dark Matter at the LHC. Eur. Phys. J. C 73(6), 2455 (2013). arXiv:1205.3169 [hep-ph]

13. J.M. Cline, K. Kainulainen, P. Scott, C. Weniger, Update on scalar singlet dark matter. Phys. Rev. D 88, 055025 (2013). arXiv:1306.4710 [hep-ph]. [Erratum: Phys. Rev. D92, no.3,039906(2015)]

14. GAMBIT, J. M. Cornell, Global fits of scalar singlet dark matter with GAMBIT. arXiv:1611.05065 [hep-ph]. [PoSICHEP2016,118(2016)]

15. R. Dick, Direct signals for electroweak singlets through the Higgs portal. arXiv: 1804.02604 [hep-ph]

16. S. Baum, M. Carena, N.R. Shah, C.E.M. Wagner, Higgs portals for thermal Dark Matter. EFT perspectives and the NMSSM'. JHEP 04, 069 (2018). arXiv:1712.09873 [hep-ph]

17. T. Cohen, J. Kearney, A. Pierce, D. Tucker-Smith, Singlet-Doublet Dark Matter. Phys. Rev. D 85, 075003 (2012). arXiv:1109.2604 [hep-ph]

18. C. Cheung, D. Sanford, Simplified models of mixed dark matter. JCAP 1402, 011 (2014). arXiv:1311.5896 [hep-ph]

19. C.E. Yaguna, Singlet-doublet dirac dark matter. Phys. Rev. D 92(11), 115002 (2015). arXiv:1510.06151 [hep-ph]

20. L. Calibbi, A. Mariotti, P. Tziveloglou, Singlet-doublet model: dark matter searches and LHC constraints. JHEP 10, 116 (2015). arXiv:1505.03867 [hep-ph]

21. Q.-F. Xiang, X.-J. Bi, P.-F. Yin, Z.-H. Yu, Exploring fermionic dark matter via higgs boson precision measurements at the circular electron positron collider. Phys. Rev. D 97(5), 055004 (2018). arXiv:1707.03094 [hep-ph]

22. S. Esch, M. Klasen, C. Yaguna, A singlet doublet dark matter model with radiative neutrino masses. arXiv:1804.03384 [hep$\mathrm{ph}]$

23. G. Arcadi, M. Dutra, P. Ghosh, M. Lindner, Y. Mambrini, M. Pierre, S. Profumo, F.S. Queiroz, The waning of the WIMP? A 
review of models, searches, and constraints. Eur. Phys. J. C 78(3), 203 (2018). arXiv:1703.07364 [hep-ph]

24. M. Escudero, A. Berlin, D. Hooper, M.-X. Lin, Toward (Finally!) ruling out $\mathrm{Z}$ and higgs mediated dark matter models. JCAP 1612, 029 (2016). arXiv: 1609.09079 [hep-ph]

25. J. Ellis, A. Fowlie, L. Marzola, M. Raidal, Statistical analyses of higgs- and Z-portal dark matter models. arXiv:1711.09912 [hep$\mathrm{ph}]$

26. G. Arcadi, Y. Mambrini, F. Richard, Z-portal dark matter. JCAP 1503, 018 (2015). arXiv:1411.2985 [hep-ph]

27. A. Angelescu, G. Arcadi, Dark matter phenomenology of SM and enlarged higgs sectors extended with vector like leptons. Eur. Phys. J. C 77(7), 456 (2017). arXiv:1611.06186 [hep-ph]

28. A. Berlin, S. Gori, T. Lin, L.-T. Wang, Pseudoscalar portal dark matter. Phys. Rev. D 92, 015005 (2015). arXiv:1502.06000 [hep$\mathrm{ph}]$

29. S. Davidson, H.E. Haber, Basis-independent methods for the two-Higgs-doublet model. Phys. Rev. D72, 035004 (2005). arXiv:hep-ph/0504050 [hep-ph]. [Erratum: Phys. Rev. D72,099902(2005)]

30. V. Zarikas, The Phase transition of the two Higgs extension of the standard model. Phys. Lett. B 384, 180-184 (1996). arXiv:hep-ph/9509338 [hep-ph]

31. A.B. Lahanas, V.C. Spanos, V. Zarikas, Charge asymmetry in two-Higgs doublet model. Phys. Lett. B 472, 119 (2000). arXiv:hep-ph/9812535 [hep-ph]

32. G. Aliferis, G. Kofinas, V. Zarikas, Efficient electroweak baryogenesis by black holes. Phys. Rev. D 91(4), 045002 (2015). arXiv: 1406.6215 [hep-ph]

33. S. Kanemura, Y. Okada, E. Senaha, C.P. Yuan, Higgs coupling constants as a probe of new physics. Phys. Rev. D 70, 115002 (2004). arXiv:hep-ph/0408364 [hep-ph]

34. D. Bečirević, E. Bertuzzo, O. Sumensari, R. Zukanovich Funchal, Can the new resonance at LHC be a CP-Odd Higgs boson?'. Phys. Lett. B 757, 261-267 (2016). arXiv:1512.05623 [hep-ph]

35. A. Barroso, P.M. Ferreira, I.P. Ivanov, R. Santos, Metastability bounds on the two Higgs doublet model. JHEP 06, 045 (2013). arXiv: 1303.5098 [hep-ph]

36. S. Davidson, G.J. Grenier, Lepton flavour violating Higgs and tau to mu gamma. Phys. Rev. D 81, 095016 (2010). arXiv:1001.0434 [hep-ph]

37. M. Baak, M. Goebel, J. Haller, A. Hoecker, D. Ludwig, K. Moenig, M. Schott, J. Stelzer, Updated status of the global electroweak fit and constraints on new physics. Eur. Phys. J. C 72, 2003 (2012). arXiv:1107.0975 [hep-ph]

38. I. Maksymyk, C.P. Burgess, D. London, Beyond S, T and U. Phys. Rev. D 50, 529-535 (1994). arXiv:hep-ph/9306267 [hep-ph]

39. H.-J. He, N. Polonsky, S.-F. Su, Extra families, Higgs spectrum and oblique corrections. Phys. Rev. D 64, 053004 (2001). arXiv:hep-ph/0102144 [hep-ph]

40. R. Barbieri, L.J. Hall, Y. Nomura, V.S. Rychkov, Supersymmetry without a Light Higgs Boson. Phys. Rev. D 75, 035007 (2007). arXiv:hep-ph/0607332 [hep-ph]

41. G.C. Branco, P.M. Ferreira, L. Lavoura, M.N. Rebelo, M. Sher, J.P. Silva, Theory and phenomenology of two-Higgs-doublet models. Phys. Rept. 516, 1-102 (2012). arXiv:1106.0034 [hep-ph]

42. E. Bertuzzo, P.A.N. Machado, M. Taoso, Di-Photon excess in the 2HDM: hasting towards the instability and the non-perturbative regime. arXiv: 1601.07508 [hep-ph]

43. P. Arnan, D. Bečirević, F. Mescia, O. Sumensari, Two Higgs doublet models and $b \rightarrow s$ exclusive decays. Eur. Phys. J. C 77(11), 796 (2017). arXiv:1703.03426 [hep-ph]

44. D. Bečirević, B. Melić, M. Patra, O. Sumensari, Seeking a CP odd Higgs boson via $h \rightarrow \eta_{c, b} \ell^{+} \ell^{-}$. Phys. Rev. D 97(1), 015008 (2018). arXiv:1705.01112 [hep-ph]
45. Gfitter Group, M. Baak, J. Cúth, J. Haller, A. Hoecker, R. Kogler, K. Mönig, M. Schott, and J. Stelzer, The global electroweak fit at NNLO and prospects for the LHC and ILC. Eur. Phys. J. C 74 (2014) 3046, arXiv:1407.3792 [hep-ph]

46. R. Enberg, P.J. Fox, L.J. Hall, A.Y. Papaioannou, M. Papucci, LHC and dark matter signals of improved naturalness. JHEP 11, 014 (2007). arXiv:0706.0918 [hep-ph]

47. F. D'Eramo, Dark matter and Higgs boson physics. Phys. Rev. D 76, 083522 (2007). arXiv:0705.4493 [hep-ph]

48. A. Joglekar, P. Schwaller, C.E.M. Wagner, Dark matter and enhanced higgs to Di-photon rate from vector-like leptons. JHEP 12, 064 (2012). arXiv:1207.4235 [hep-ph]

49. ATLAS, M. Aaboud et al., Search for additional heavy neutral Higgs and gauge bosons in the ditau final state produced in $36 \mathrm{fb}^{-1}$ of pp collisions at $\sqrt{s}=13 \mathrm{TeV}$ with the ATLAS detector. JHEP 01 (2018) 055, arXiv: 1709.07242 [hep-ex]

50. CMS Collaboration, Search for a neutral MSSM Higgs boson decaying into $\tau \tau$ with $12.9 \mathrm{fb}^{-1}$ of data at $\sqrt{s}=13 \mathrm{TeV}$. CMS-PAS-HIG-16-037, CERN, Geneva, 2016. https://cds.cern. $\mathrm{ch} / \mathrm{record} / 2231507$

51. CMS, V. Khachatryan et al., Search for neutral MSSM Higgs bosons decaying into a pair of bottom quarks. JHEP 11 (2015) 071. arXiv:1506.08329 [hep-ex]

52. CMS Collaboration, Search for a narrow heavy decaying to bottom quark pairs in the $13 \mathrm{TeV}$ data sample. CMS-PAS-HIG-16025, CERN, Geneva, 2016. https://cds.cern.ch/record/2204928

53. ATLAS, M. Aaboud et al., Evidence for the $H \rightarrow b \bar{b}$ decay with the ATLAS detector. JHEP 12 (2017) 024. arXiv: 1708.03299 [hep-ex]

54. CMS, A. M. Sirunyan et al., Search for a light pseudoscalar Higgs boson produced in association with bottom quarks in pp collisions at $\sqrt{s}=8 \mathrm{TeV}$. JHEP 11 (2017) 010. arXiv:1707.07283 [hep-ex]

55. ATLAS, M. Aaboud et al., Search for Heavy Higgs Bosons $A / H$ Decaying to a Top Quark Pair in $p p$ Collisions at $\sqrt{s}=8 \mathrm{TeV}$ with the ATLAS Detector. Phys. Rev. Lett. 119(19), 191803 (2017). arXiv:1707.06025 [hep-ex]

56. CMS Collaboration, Measurements of properties of the Higgs boson and search for an additional resonance in the four-lepton final state at sqrt(s) $=13 \mathrm{TeV}$. CMS-PAS-HIG-16-033, CERN, Geneva, 2016. https://cds.cern.ch/record/2204926

57. CMS Collaboration, Search for new diboson resonances in the dilepton + jets final state at $\sqrt{s}=13 \mathrm{TeV}$ with 2016 data. CMS-PAS-HIG-16-034, CERN, Geneva, 2017. https://cds.cern. $\mathrm{ch} /$ record/2243295

58. ATLAS, M. Aaboud et al., Searches for heavy diboson resonances in $p p$ collisions at $\sqrt{s}=13 \mathrm{TeV}$ with the ATLAS detector. JHEP 09, 173 (2016). arXiv:1606.04833 [hep-ex]

59. ATLAS, M. Aaboud et al., Searches for heavy $Z Z$ and $Z W$ resonances in the $\ell \ell q q$ and $v v q q$ final states in $p p$ collisions at $\sqrt{s}=13 \mathrm{TeV}$ with the ATLAS detector. JHEP 03, 009 (2018). arXiv:1708.09638 [hep-ex]

60. CMS Collaboration, Search for high mass Higgs to WW with fully leptonic decays using 2015 data. CMS-PAS-HIG-16-023, CERN, Geneva, 2016. https://cds.cern.ch/record/2205151

61. ATLAS, M. Aaboud et al., Search for $W W / W Z$ resonance production in $\ell v q q$ final states in $p p$ collisions at $\sqrt{s}=13 \mathrm{TeV}$ with the ATLAS detector. arXiv:1710.07235 [hep-ex]

62. ATLAS, M. Aaboud et al., Search for heavy resonances decaying into $W W$ in the $e v \mu v$ final state in $p p$ collisions at $\sqrt{s}=13$ $\mathrm{TeV}$ with the ATLAS detector. Eur. Phys. J. C 78(1), 24 (2018). arXiv:1710.01123 [hep-ex]

63. ATLAS, M. Aaboud et al., Search for new phenomena in highmass diphoton final states using $37 \mathrm{fb}^{-1}$ of proton-proton collisions collected at $\sqrt{s}=13 \mathrm{TeV}$ with the ATLAS detector. Phys. Lett. B 775, 105-125 (2017). arXiv:1707.04147 [hep-ex] 
64. ATLAS Collaboration, Search for Higgs boson pair production in the final state of $\gamma \gamma W W^{*}(\rightarrow l v j j)$ using $13.3 \mathrm{fb}^{-1}$ of $p p$ collision data recorded at $\sqrt{s}=13 \mathrm{TeV}$ with the ATLAS detector. ATLASCONF-2016-071, CERN, Geneva, Aug, 2016. http://cds.cern.ch/ record $/ 2206222$

65. CMS Collaboration, Search for resonant pair production of Higgs bosons decaying to two bottom quark-antiquark pairs in protonproton collisions at $13 \mathrm{TeV}$. CMS-PAS-HIG-16-002, CERN, Geneva, 2016. https://cds.cern.ch/record/2141024

66. CMS Collaboration, Search for $\mathrm{H}(\mathrm{bb}) \mathrm{H}(\gamma \gamma)$ decays at $13 \mathrm{TeV}$. CMS-PAS-HIG-16-032, CERN, Geneva, 2016. https://cds.cern. $\mathrm{ch} /$ record/2207960

67. Search for Higgs boson pair production in the $b \bar{b} \gamma \gamma$ final state using pp collision data at $\sqrt{s}=13 \mathrm{TeV}$ with the ATLAS detector. ATLAS-CONF-2016-004, CERN, Geneva, Mar, 2016. http://cds. cern.ch/record/2138949

68. CMS, A. M. Sirunyan et al., Search for Higgs boson pair production in events with two bottom quarks and two tau leptons in proton proton collisions at $\sqrt{s}=13 \mathrm{TeV}$. Phys. Lett. B 778, 101-127 (2018). arXiv:1707.02909 [hep-ex]

69. CMS, A. M. Sirunyan et al., Search for resonant and nonresonant Higgs boson pair production in the $b \bar{b} \ell v \ell v$ final state in proton-proton collisions $\sqrt{s}=1 \mathrm{TeV}$. JHEP 01, 054 (2018). arXiv: 1708.04188 [hep-ex]

70. Search for a CP-odd Higgs boson decaying to $\mathrm{Zh}$ in pp collisions at $\sqrt{s}=13 \mathrm{TeV}$ with the ATLAS detector. ATLAS-CONF2016-015, CERN, Geneva, Mar, 2016. http://cds.cern.ch/record/ 2141003

71. ATLAS, G. Aad et al., Search for a CP-odd Higgs boson decaying to $\mathrm{Zh}$ in pp collisions at $\sqrt{s}=8 \mathrm{TeV}$ with the ATLAS detector. Phys. Lett. B 744, 163-183 (2015). arXiv:1502.04478 [hep-ex]

72. CMS, V. Khachatryan et al., Searches for a heavy scalar boson $\mathrm{H}$ decaying to a pair of $125 \mathrm{GeV}$ Higgs bosons hh or for a heavy pseudoscalar boson A decaying to $\mathrm{Zh}$, in the final states with $h \rightarrow \tau \tau$. Phys. Lett. B 755, 217-244 (2016). arXiv:1510.01181 [hep-ex]

73. CMS, V. Khachatryan et al., Search for a pseudoscalar boson decaying into a $\mathrm{Z}$ boson and the $125 \mathrm{GeV}$ Higgs boson in $\ell^{+} \ell^{-} b \bar{b}$ final states. Phys. Lett. B 748, 221-243 (2015). arXiv:1504.04710 [hep-ex]

74. CMS, V. Khachatryan et al., Search for neutral resonances decaying into a $\mathrm{Z}$ boson and a pair of $\mathrm{b}$ jets or $\tau$ leptons. Phys. Lett. B 759, 369-394 (2016). arXiv:1603.02991 [hep-ex]

75. ATLAS, G. Aad et al., Measurements of the Higgs boson production and decay rates and coupling strengths using pp collision data at $\sqrt{s}=7$ and $8 \mathrm{TeV}$ in the ATLAS experiment. Eur. Phys. J. C 76(1), 6 (2016). arXiv:1507.04548 [hep-ex]

76. CMS, V. Khachatryan et al., Precise determination of the mass of the Higgs boson and tests of compatibility of its couplings with the standard model predictions using proton collisions at 7 and $8 \mathrm{TeV}$. Eur. Phys. J. C 75(5), 212 (2015). arXiv:1412.8662 [hep-ex]

77. ATLAS, CMS, G. Aad et al., Measurements of the Higgs boson production and decay rates and constraints on its couplings from a combined ATLAS and CMS analysis of the LHC pp collision data at $\sqrt{s}=7$ and 8 TeV. JHEP 08, 045 (2016). arXiv:1606.02266 [hep-ex]

78. H. Bélusca-Maïto, A. Falkowski, D. Fontes, J.C. Romão, J.P. Silva, Higgs EFT for 2HDM and beyond. Eur. Phys. J. C 77(3), 176 (2017). arXiv:1611.01112 [hep-ph]

79. M. Bauer, M. Klassen, V. Tenorth, Universal Properties of Pseudoscalar Mediators. arXiv:1712.06597 [hep-ph]

80. J. Gu, H. Li, Z. Liu, S. Su, W. Su, Learning from higgs physics at future higgs factories. JHEP 12, 153 (2017). arXiv:1709.06103 [hep-ph]
81. P.M. Ferreira, J.F. Gunion, H.E. Haber, R. Santos, Probing wrongsign Yukawa couplings at the LHC and a future linear collider. Phys. Rev. D 89(11), 115003 (2014). arXiv:1403.4736 [hep-ph]

82. D. Fontes, J.C. Romão, J.P. Silva, A reappraisal of the wrongsign $h b \bar{b}$ coupling and the study of $h \rightarrow Z \gamma$. Phys. Rev. D 90(1), 015021 (2014). arXiv:1406.6080 [hep-ph]

83. P.M. Ferreira, R. Guedes, J.F. Gunion, H.E. Haber, M.O.P. Sampaio, R. Santos, The CP-conserving 2HDM after the $8 \mathrm{TeV}$ run. In Proceedings, 22nd International Workshop on Deep-Inelastic Scattering and Related Subjects (DIS 2014): Warsaw, Poland, April 28-May 2, 2014. 2014. arXiv:1407.4396 [hep-ph]

84. CMS, V. Khachatryan et al., Search for light bosons in decays of the $125 \mathrm{GeV}$ Higgs boson in proton-proton collisions at $\sqrt{s}=8$ TeV. JHEP 10, 076 (2017). arXiv:1701.02032 [hep-ex]

85. M. Bauer, U. Haisch, F. Kahlhoefer, Simplified dark matter models with two Higgs doublets: I. Pseudoscalar mediators. JHEP 05, 138 (2017). arXiv:1701.07427 [hep-ph]

86. D. Goncalves, P.A.N. Machado, J.M. No, Simplified models for dark matter face their consistent completions. Phys. Rev. D 95(5), 055027 (2017). arXiv:1611.04593 [hep-ph]

87. LEP, DELPHI, OPAL, ALEPH, L3, G. Abbiendi et al., Search for charged higgs bosons: combined results using LEP data. Eur. Phys. J. C 73,2463 (2013). arXiv:1301.6065 [hep-ex]

88. ATLAS, G. Aad et al., Search for a light charged Higgs boson in the decay channel $H^{+} \rightarrow c \bar{s}$ in $t \bar{t}$ events using pp collisions at $\sqrt{s}=7 \mathrm{TeV}$ with the ATLAS detector. Eur. Phys. J. C 73(6), 2465 (2013). arXiv:1302.3694 [hep-ex]

89. ATLAS, G. Aad et al., Search for charged Higgs bosons decaying via $H^{ \pm} \rightarrow \tau^{ \pm} v$ in fully hadronic final states using $p p$ collision data at $\sqrt{s}=8 \mathrm{TeV}$ with the ATLAS detector. JHEP 03, 088 (2015). arXiv:1412.6663 [hep-ex]

90. CMS, V. Khachatryan et al., Search for a charged Higgs boson in pp collisions at $\sqrt{s}=8 \mathrm{TeV}$. JHEP 11, 018 (2015). arXiv: 1508.07774 [hep-ex]

91. CMS, V. Khachatryan et al., Search for a light charged Higgs boson decaying to $\bar{c} \bar{s}$ in pp collisions at $\sqrt{s}=8 \mathrm{TeV}$. JHEP 12, 178 (2015). arXiv:1510.04252 [hep-ex]

92. A. Arbey, F. Mahmoudi, O. Stal, T. Stefaniak, Status of the charged higgs boson in two higgs doublet models. Eur. Phys. J. C 78(3), 18 (2018). arXiv:1706.07414 [hep-ph]

93. ATLAS, M. Aaboud et al., Search for charged Higgs bosons produced in association with a top quark and decaying via $H^{ \pm} \rightarrow \tau \nu$ using $p p$ collision data recorded at $\sqrt{s}=13 \mathrm{TeV}$ by the ATLAS detector. Phys. Lett. B 759, 555-574 (2016). arXiv:1603.09203 [hep-ex]

94. ATLAS Collaboration, Search for charged Higgs bosons in the $H^{ \pm} \rightarrow t b$ decay channel in $p p$ collisions at $\sqrt{s}=13 \mathrm{TeV}$ using the ATLAS detector. ATLAS-CONF-2016-089, CERN, Geneva, Aug, 2016. https://cds.cern.ch/record/2206809

95. CMS Collaboration, Search for charged Higgs bosons with the $\mathrm{H}^{ \pm} \rightarrow \tau^{ \pm} v_{\tau}$ decay channel in the fully hadronic final state at $\sqrt{s}=13$ TeV. CMS-PAS-HIG-16-031, CERN, Geneva, 2016. https://cds.cern.ch/record/2223865

96. HFLAV, Y. Amhis et al., Averages of $b$-hadron, $c$-hadron, and $\tau$ lepton properties as of summer 2016. Eur. Phys. J. C 77(12), 895 (2017). arXiv:1612.07233 [hep-ex]

97. M. Misiak, M. Steinhauser, Weak radiative decays of the B meson and bounds on $M_{H^{ \pm}}$in the Two-Higgs-Doublet Model. Eur. Phys. J. C 77(3), 201 (2017). arXiv:1702.04571 [hep-ph]

98. LHC Higgs Cross Section Working Group, D. de Florian et al., Handbook of LHC higgs cross sections: 4. deciphering the nature of the higgs sector. arXiv:1610.07922 [hep-ph]

99. R.V. Harlander, S. Liebler, H. Mantler, SusHi: A program for the calculation of Higgs production in gluon fusion and bottom-quark annihilation in the Standard Model and the MSSM. Comput. Phys. Commun. 184, 1605-1617 (2013). arXiv:1212.3249 [hep-ph] 
100. A. Djouadi, L. Maiani, G. Moreau, A. Polosa, J. Quevillon, V. Riquer, The post-Higgs MSSM scenario: Habemus MSSM? Eur. Phys. J. C 73, 2650 (2013). arXiv:1307.5205 [hep-ph]

101. A. Djouadi, L. Maiani, A. Polosa, J. Quevillon, V. Riquer, Fully covering the MSSM Higgs sector at the LHC. JHEP 06, 168 (2015). arXiv: 1502.05653 [hep-ph]

102. P. Gondolo, G. Gelmini, Cosmic abundances of stable particles: Improved analysis. Nucl. Phys. B 360, 145-179 (1991)

103. J. Edsjo, P. Gondolo, Neutralino relic density including coannihilations. Phys. Rev. D 56, 1879-1894 (1997). arXiv:hep-ph/9704361 [hep-ph]

104. G. Belanger, F. Boudjema, A. Pukhov, A. Semenov, MicrOMEGAs 2.0: A Program to calculate the relic density of dark matter in a generic model. Comput. Phys. Commun. 176, 367-382 (2007). arXiv:hep-ph/0607059 [hep-ph]

105. A. Alloul, N.D. Christensen, C. Degrande, C. Duhr, B. Fuks, FeynRules 2.0 - a complete toolbox for tree-level phenomenology. Comput. Phys. Commun. 185, 2250-2300 (2014). arXiv:1310.1921 [hep-ph]

106. S. Banerjee, S. Matsumoto, K. Mukaida, Y.-L.S. Tsai, WIMP dark matter in a well-tempered regime: a case study on singlet-doublets fermionic WIMP. JHEP 11, 070 (2016). arXiv:1603.07387 [hep$\mathrm{ph}]$

107. A. Bharucha, F. Brümmer, R. Ruffault, Well-tempered n-plet dark matter. JHEP 09, 160 (2017). arXiv: 1703.00370 [hep-ph]

108. K. Griest, Calculations of rates for direct detection of neutralino dark matter. Phys. Rev. Lett. 61, 666-669 (1988)

109. M. Drees, M. Nojiri, Neutralino-nucleon scattering revisited'. Phys. Rev. D 48, 3483-3501 (1993). arXiv:hep-ph/9307208 [hep$\mathrm{ph}]$

110. XENON, E. Aprile et al., First dark matter search results from the XENON1T experiment. arXiv:1705.06655 [astro-ph.CO]

111. LUX, D. S. Akerib et al., Results from a search for dark matter in the complete LUX exposure. Phys. Rev. Lett. 118, 021303 (2017). arXiv:1608.07648 [astro-ph.CO]

112. PandaX-II, A. Tan et al., Dark matter results from first 98.7 days of data from the PandaX-II Experiment. Phys. Rev. Lett. 117, 121303 (2016). arXiv: 1607.07400 [hep-ex]

113. LUX, LZ, M. Szydagis, The Present and Future of Searching for Dark Matter with LUX and LZ. PoS ICHEP2016, 220 (2016). arXiv:1611.05525 [astro-ph.CO]

114. XENON, E. Aprile et al., Physics reach of the XENON1T dark matter experiment. JCAP 1604(04), 027 (2016). arXiv:1512.07501 [physics.ins-det]

115. DARWIN, J. Aalbers et al., DARWIN: towards the ultimate dark matter detector. JCAP 1611, 017 (2016). arXiv:1606.07001 [astro-ph.IM]

116. LUX, D. S. Akerib et al., Limits on spin-dependent WIMPnucleon cross section obtained from the complete LUX exposure", Phys. Rev. Lett. 118(25), 251302 (2017). arXiv: 1705.03380 [astro-ph.CO]

117. C. Cheung, L.J. Hall, D. Pinner, J.T. Ruderman, Prospects and Blind spots for neutralino dark matter. JHEP 05, 100 (2013). arXiv:1211.4873 [hep-ph]

118. P. Huang, C.E.M. Wagner, Blind spots for neutralino dark matter in the MSSM with an intermediate $m_{A}$. Phys. Rev. D 90(1), 015018 (2014). arXiv:1404.0392 [hep-ph]

119. A. Choudhury, K. Kowalska, L. Roszkowski, E.M. Sessolo, A.J. Williams, Blind spots for direct detection with simplified DM models and the LHC. Universe 3(2), 41 (2017). arXiv:1705.04230 [hep-ph]

120. J. Hisano, K. Ishiwata, N. Nagata, Gluon contribution to the dark matter direct detection. Phys. Rev. D 82, 115007 (2010). arXiv:1007.2601 [hep-ph]
121. J. Hisano, K. Ishiwata, N. Nagata, A complete calculation for direct detection of Wino dark matter. Phys. Lett. B 690, 311-315 (2010). arXiv:1004.4090 [hep-ph]

122. J. Hisano, K. Ishiwata, N. Nagata, T. Takesako, Direct detection of electroweak-interacting dark matter. JHEP 07, 005 (2011). arXiv:1104.0228 [hep-ph]

123. S. Ipek, D. McKeen, A.E. Nelson, A renormalizable model for the galactic center gamma ray excess from dark matter annihilation. Phys. Rev. D 90(5), 055021 (2014). arXiv:1404.3716 [hep-ph]

124. G. Arcadi, M. Lindner, F.S. Queiroz, W. Rodejohann, S. Vogl, Pseudoscalar mediators: a WIMP model at the neutrino floor. arXiv:1711.02110 [hep-ph]

125. I.W. Sanderson, N.F. Bell, G. Busoni, Loop effects in direct detection. arXiv:1803.01574 [hep-ph]

126. J. Guo, J. Li, T. Li, A.G. Williams, NMSSM explanations of the Galactic center gamma ray excess and promising LHC searches. Phys. Rev. D 91(9), 095003 (2015). arXiv:1409.7864 [hep-ph]

127. C. Cheung, M. Papucci, D. Sanford, N.R. Shah, K.M. Zurek, NMSSM Interpretation of the Galactic Center Excess. Phys. Rev. D 90(7), 075011 (2014). arXiv:1406.6372 [hep-ph]

128. DES, Fermi-LAT, A. Albert et al., Searching for dark matter annihilation in recently discovered milky way satellites with fermiLAT. Astrophys. J. 834(2), 110 (2017). arXiv:1611.03184 [astroph.HE]

129. L. J. Chang, M. Lisanti, S. Mishra-Sharma, A search for dark matter annihilation in the milky way halo. arXiv:1804.04132 [astroph.CO]

130. Particle Data Group, C. Patrignani et al., Review of particle physics. Chin. Phys. C 40(10), 100001 (2016)

131. ATLAS, M. Aaboud et al., Search for electroweak production of supersymmetric particles in final states with two or three leptons at $\sqrt{s}=13 \mathrm{TeV}$ with the ATLAS detector. arXiv:1803.02762 [hep-ex]

132. CMS, A. M. Sirunyan et al., Combined search for electroweak production of charginos and neutralinos in proton-proton collisions at $\sqrt{s}=13 \mathrm{TeV}$. JHEP 03, 160 (2018) arXiv:1801.03957 [hep-ex]

133. L. Calibbi, J.M. Lindert, T. Ota, Y. Takanishi, LHC tests of light neutralino dark matter without light sfermions. JHEP 11, 106 (2014). arXiv:1410.5730 [hep-ph]

134. CMS, V. Khachatryan et al., Searches for electroweak production of charginos, neutralinos, and sleptons decaying to leptons and W, Z, and Higgs bosons in pp collisions at 8 TeV. Eur. Phys. J. C 74(9), 3036 (2014). arXiv:1405.7570 [hep-ex]

135. ATLAS, G. Aad et al., Search for direct production of charginos and neutralinos in events with three leptons and missing transverse momentum in $\sqrt{s}=8 \mathrm{TeV} p p$ collisions with the ATLAS detector. JHEP 04 (2014) 169, arXiv:1402.7029 [hep-ex]

136. ATLAS, M. Aaboud et al., Search for dark matter and other new phenomena in events with an energetic jet and large missing transverse momentum using the ATLAS detector. JHEP 01, 126 (2018). arXiv:1711.03301 [hep-ex]

137. CMS, A. M. Sirunyan et al., Search for new physics in final states with an energetic jet or a hadronically decaying $W$ or $Z$ boson and transverse momentum imbalance at $\sqrt{s}=13 \mathrm{TeV}$. Phys. Rev. D97(9), 092005 (2018). arXiv:1712.02345 [hep-ex]

138. S. Banerjee, D. Barducci, G. Bélanger, B. Fuks, A. Goudelis, B. Zaldivar, Cornering pseudoscalar-mediated dark matter with the LHC and cosmology. JHEP 07, 080 (2017). arXiv:1705.02327 [hep-ph]

139. DELPHI, J. Abdallah et al., Searches for supersymmetric particles in e+ e- collisions up to $208-\mathrm{GeV}$ and interpretation of the results within the MSSM. Eur. Phys. J. C 31, 421-479 (2003). arXiv:hep-ex/0311019 [hep-ex] 
140. M. Cirelli, A. Strumia, M. Tamburini, Cosmology and astrophysics of minimal dark matter. Nucl. Phys. B 787, 152-175 (2007). arXiv:0706.4071 [hep-ph]

141. M. Cirelli, A. Strumia, Minimal dark matter: model and results. New J. Phys. 11, 105005 (2009). arXiv:0903.3381 [hep-ph]

142. M. Cirelli, T. Hambye, P. Panci, F. Sala, M. Taoso, Gamma ray tests of minimal dark matter. JCAP 1510(10), 026 (2015). arXiv:1507.05519 [hep-ph]

143. C. Garcia-Cely, J. Heeck, Phenomenology of left-right symmetric dark matter. arXiv:1512.03332 [hep-ph]. [JCAP1603,021(2016)]

144. L. Lopez Honorez, M.H.G. Tytgat, P. Tziveloglou, B. Zaldivar, On minimal dark matter coupled to the higgs. JHEP 04, 011 (2018). arXiv:1711.08619 [hep-ph]

145. M. Beneke, C. Hellmann, P. Ruiz-Femenia, Non-relativistic pair annihilation of nearly mass degenerate neutralinos and charginos III. Computation of the Sommerfeld enhancements. JHEP 05, 115 (2015). arXiv:1411.6924 [hep-ph]

146. M. Beneke, A. Bharucha, F. Dighera, C. Hellmann, A. Hryczuk, S. Recksiegel, P. Ruiz-Femenia, Relic density of wino-like dark matter in the MSSM. JHEP 03, 119 (2016). arXiv:1601.04718 [hep-ph]

147. M. Beneke, A. Bharucha, A. Hryczuk, S. Recksiegel, P. RuizFemenia, The last refuge of mixed wino-Higgsino dark matter. JHEP 01, 002 (2017). arXiv:1611.00804 [hep-ph]

148. J. March-Russell, S.M. West, D. Cumberbatch, D. Hooper, Heavy dark matter through the higgs portal. JHEP 07, 058 (2008). arXiv:0801.3440 [hep-ph]
149. P.F. Bedaque, M.I. Buchoff, R.K. Mishra, Sommerfeld enhancement from Goldstone pseudo-scalar exchange. JHEP 11, 046 (2009). arXiv:0907.0235 [hep-ph]

150. Z.-P. Liu, Y.-L. Wu, Y.-F. Zhou, Sommerfeld enhancements with vector, scalar and pseudoscalar force-carriers. Phys. Rev. D 88, 096008 (2013). arXiv:1305.5438 [hep-ph]

151. G. Ovanesyan, T.R. Slatyer, I.W. Stewart, Heavy dark matter annihilation from effective field theory. Phys. Rev. Lett. 114(21), 211302 (2015). arXiv:1409.8294 [hep-ph]

152. M. Baumgart, I.Z. Rothstein, V. Vaidya, Calculating the annihilation rate of weakly interacting massive particles. Phys. Rev. Lett. 114, 211301 (2015). arXiv:1409.4415 [hep-ph]

153. M. Bauer, T. Cohen, R.J. Hill, M.P. Solon, Soft collinear effective theory for heavy WIMP annihilation. JHEP 01, 099 (2015). arXiv:1409.7392 [hep-ph]

154. C. Garcia-Cely, A. Ibarra, A.S. Lamperstorfer, M.H.G. Tytgat, Gamma-rays from heavy minimal dark matter. JCAP 1510(10), 058 (2015). arXiv:1507.05536 [hep-ph]

155. M. Baumgart, V. Vaidya, Semi-inclusive wino and higgsino annihilation to $L L^{\prime}$. JHEP 03, 213 (2016). arXiv: 1510.02470 [hep-ph]

156. E.J. Chun, S. Jung, J.-C. Park, Very degenerate higgsino dark matter. JHEP 01, 009 (2017). arXiv:1607.04288 [hep-ph] 